\title{
Adaptive hybrid position/force control of dual-arm cooperative manipulators with uncertain dynamics and closed-chain kinematics
}

\author{
Yi Ren ${ }^{\mathrm{a}}$, Zhengsheng Chen ${ }^{\mathrm{b}}$, Yechao Liu ${ }^{\mathrm{a}}$, Yikun $\mathrm{Gu}^{\mathrm{a}, *}$, Minghe Jin ${ }^{\mathrm{a}}$, \\ Hong $\mathrm{Liu}^{\mathrm{a}}$ \\ a State Key Laboratory of Robotics and System, Harbin Institute of Technology, Harbin, China \\ ${ }^{\mathrm{b}}$ Mechatronic Institute, Zhejiang Sci-Tech University, Hangzhou, China
}

Received 15 January 2017; received in revised form 6 August 2017; accepted 21 September 2017

Available online 28 September 2017

\begin{abstract}
In this paper, a novel adaptive control for dual-arm cooperative manipulators is proposed to accomplish the hybrid position/force tracking in the presence of dynamic and closed-chain kinematic uncertainties. Self-convergent parameter estimation of the grasped object's centre of mass and contact force estimation are incorporated into this systematic scheme. Moreover, internal force and contact force tracking objectives are achieved simultaneously by incorporating into the position tracking formula with proper null-space projection and rotation transformation. Noisy force derivative signals are not required. This adaptive controller is mathematically derived based on Lyapunov stability analysis. Three sets of simulations corresponding to three different situations are presented to verify the effectiveness and superiority of the proposed controller.
\end{abstract}

(C) 2017 The Franklin Institute. Published by Elsevier Ltd. All rights reserved.

\section{Introduction}

Dual-arm cooperative manipulator system has drawn increased attention of numerous researchers in recent years due to its great carrying capacity and versatility in the bimanual tasks

\footnotetext{
* Corresponding author.

E-mail address: yikungu_hitdlr@163.com (Y. Gu).
} 
it can perform. These cooperative tasks can be generally subdivided into asymmetric one and symmetric one [1]. In asymmetric bimanual tasks, each manipulator performs a different role to achieve a desired task, e.g., opening a jar or writing a handy note. In symmetric bimanual tasks, both arms play the same role: in an out-of-phase manner, e.g., rope climbing, or in an in-phase manner, e.g., grasping a common object [2]. Two manipulators cooperate with each other to accomplish these tasks. According to the coupling strength, dual-arm cooperation can be classified into two categories: loose cooperation and tight cooperation [3]. Unlike the former one in which the manipulators are only weakly coupled by kinematic constraint, tight cooperation means that two manipulators physically interact through a common grasped object, for example, dual-arm cooperative system is employed to manipulate an object, e.g., a welding pistol, to execute preassigned welding task. Control of a tightly cooperated dualarm system which this paper considers has great potential to utilize various tools. However, the advantage comes at the cost of an increased complexity for controlling the manipulator ensemble. Since the dual-arm manipulators and the tightly grasped object form a closed kinematic chain, a set of kinematic and dynamic constraints are imposed on the motion of the interconnected system, then degree of freedom of the whole system will degrade and internal forces are generated. These forces must be controlled appropriately, or losing control of them may lead to grasp failure or unrecoverable damage to the end-effector or the object.

To keep safety and controllable interaction, impedance control and hybrid position/force control (HPF) [4] are two schemes extensively used in the dual-arm cooperative manipulation tasks. Although approaches of internal impedance [5], object impedance [6] and combined multi-layer impedance $[7,8]$ are proposed to achieve desired interaction in the end-effector level and object level, and can be applied without any switching procedure between contact and non-contact case, lack of precision in controlling position of the grasped object and the contact force as compared to hybrid position/force control may limit their application in some situations like surgery, manufacturing, precision welding, etc. [9]. A typical HPF control is the architecture proposed by Wen and Kreutz-Delgado [10] in which tracking control of contact force and position are decoupled and both achieved. Various motion control strategies developed for single manipulator can be simply incorporated into this scheme.

The manipulator dynamics can be linearly expressed by some parameters, i.e., mass, inertial, link length, centre of mass, etc. Uncertainties in these parameters are often called as dynamic uncertainties. To maintain high tracking performance when dynamic uncertainties exist in cooperative robotic system, Walker [11] incorporated adaptive mechanism into the multi-manipulator coordination in which joint acceleration signals are needed. This restriction was later relaxed in [12]. Pagilla and Tomizuka [13] then presented an adaptive hybrid position/force control strategy. Both object's motion and contact force between the grasped object and the constraint surface are controlled in a designed manner. Furthermore, unstructured uncertainties, which exist ubiquitously in system dynamics, are often caused by unmodelled terms, such as nonlinear friction, compliance in gearing and external disturbances, etc. To deal with not only dynamic uncertainties but also unstructured uncertainties, Gueaieb et al. [14] and Lian et al. [15] introduced neural network and fuzzy system into the control of cooperative manipulators respectively. Although both the position and internal force tracking are handled simultaneously, the grasped object interacting with environment is not considered. To simultaneously achieve the multiple objectives including tracking control of position/contact force/internal force and further optimization, a systematic adaptive scheme based on virtual decomposition is proposed in [16]. 
Although the controllers mentioned above can cope with dynamic uncertainties of the manipulator and object, the closed-chain kinematics is assumed to be known exactly. A problem is that stability of the cooperative controller is very sensitive to kinematic uncertainties of the interconnected system. In tight cooperation, since the manipulators rigidly contact with the object, small kinematic error may lead to large tracking error and losing control of the internal force.

The kinematic uncertainties in dual-arm system have not been extensively discussed and studied due to the complexity and diversity of sources. If interaction between the grasped object and unknown environment is also considered, kinematic uncertainties in this closed chain can be translated into: (1) geometrical uncertainty of the object (grasp matrix uncertainty); (2) kinematic uncertainty of the manipulator ensemble (robot Jacobian matrix uncertainty); (3) geometrical uncertainty of the contact surface (constraint Jacobian uncertainty [17-19]); (4) uncertainty of relative kinematics between the manipulator ensemble (relative Jacobian uncertainty [20]).

When dual-arm system grasps tools with different sizes or different grasp posture/point, the geometrical uncertainty of the tool would lead to the variation of the grasp matrix, and further the closed-chain kinematics of the interconnected system. Knowable decouple of the grasp force cannot be obtained, let alone the internal force tracking. Furthermore, to enable the system to dexterously grasp various tools, general end-effectors, i.e., dexterous hand or anthropomorphic prosthetic hand, are usually mounted at the end of the manipulators. Kinematics (Jacobian matrix) and dynamics of the manipulators themselves are then uncertain and cannot be exactly known due to the arbitrary grasp posture/point. Therefore, these two kinds of kinematic uncertainties are the most urgent to be addressed when achieving adaptation to different tools.

When coping with the unknown object, some researches often assume that the object's centre of mass (COM) is known but dynamic parameters are unknown. Jafari and Ryu [21] proposed a general hybrid position/force control scheme to cooperate manipulators handling an unknown object in contact with environment. This scheme is robust with varying system dynamics and does not use any derivative signal of contact force or internal force. Unknown $\mathrm{COM}$ of the object is further considered in [22]. An observer-based adaptive controller is proposed for cooperative robotic system handling an object with unknown geometry, COM, and inertial. The grasped object is handled to move in free space or interact with environment. Both two schemes rely on the assumption that kinematics of the manipulators is known.

Cheah et al. [23] and Wang and Xie [24] respectively proposed globally convergent adaptive hybrid position/force controllers for robotic arm and free-flying manipulators to simultaneously deal with the uncertain kinematics and dynamics. This approach aims at single manipulator system and cannot be simply extended to the case of interconnected cooperative system for its complexity and the extra internal force control objective. Furthermore, position of the object's COM cannot be obtained by the vision sensors. Zhao and Cheah [25] presented a neural network controller for the multi-fingered robot hand to address the kinematic/dynamic uncertainties with internal force simultaneously controlled. Nevertheless, desired interaction behaviour between grasped object and environment cannot be achieved. In addition, dualarm (multi-arm) system is much different from multi-fingered system in some key aspects as stated in [26]. For dual-arm (multi-arm) system, the kinematic chain is totally determined and the grasp type allows bilateral force transmission, which cannot be applied to multi-fingered system [27]. This difference leads to the significant difference of force analysis and further of the model-based controller design. 
It should be noted that most of the schemes presented in the literature address one or two kinds of uncertainties or achieve part of the control objectives, it still has not found a solution to systematically control a dual-arm manipulator system with uncertain kinematics/dynamics manipulating a geometrically/dynamically uncertain object and interacting with environment in a desired manner. A dual-arm robotic system which can successfully complete a certain task in the presence of dynamic and kinematic uncertainties can imitate the human strategy to a great extent, which means the robotic system can grasp and manipulate various tools without precise pre-calibration of the general end-effectors/tools. Thus, customizing certain end-effectors for certain tools can then be avoided. This anthropomorphic characteristic endows the dual-arm manipulators with broader application in the field of industry production and space exploration.

Hence in this paper, to address the hybrid position/force tracking problem when uncertainties exist in both dynamics and closed-chain kinematics, we propose a novel adaptive controller for dual-arm cooperative manipulators handling an unknown object in contact with environment. According to different sources, two kinds of kinematic uncertainties are discussed and coped with separately. Dynamic uncertainties are addressed based on the complete dynamical equation of the interconnected system. This adaptive controller is designed and analysed based on Lyapunov stability theorem. Three sets of simulations are implemented to present the superiority of the proposed controller in the free-space motion control, HPF control with flat constraint surface and curved surface.

\section{Problem formulation}

Consider two cooperative manipulators tightly grasping a common unknown object whose end tip contacts with rigid environment (or rigid contact/constraint surface), as shown in Fig. 1. All the poses and forces are represented with respect to the world frame.

Assumption 1. The unknown object is rigid and the dual-arm system grasps the object tightly so that there is no relative motion between the end-effectors and the object. Grasp strategy and planning will not be discussed here.

Assumption 2. Poses of the object's end tip and two end-effectors can be sensed by a vision system and fed back to the control system in real time.

Assumption 3. Environment geometry is known. No plastic deformation will be produced by the contact force.

\subsection{Kinematics and dynamics of the interconnected system}

\subsubsection{Kinematics}

Denoting $x_{e}=\left[\begin{array}{cc}x_{e 1}^{T} & x_{e 2}^{T}\end{array}\right]^{T} \in R^{2 m \times 1}$ as the pose vector of the two end-effectors and it is related to joint angle velocities by

$\dot{x}_{e}=J_{D} \dot{q}_{D}$

where $q_{D}=\left[q_{1}^{T}, q_{2}^{T}\right]^{T} \in R^{\left(n_{1}+n_{2}\right) \times 1}$ and $q_{i} \in R^{n_{i} \times 1}$ for $i=1,2$ is the joint angle vector of the $i$ th manipulator; $J_{D}=\operatorname{blockdiag}\left[J_{1}, J_{2}\right] \in R^{2 m \times\left(n_{1}+n_{2}\right)}$ where $J_{i}$ with $m \leq n_{i}$ denotes the Jacobian matrix of the $i$ th manipulator. Eq. (1) can be expressed linearly in a set of kinematic parameters $\theta_{k}=\left[\theta_{k 1}, \theta_{k 2}, \ldots, \theta_{k j}\right]^{T} \in R^{j}$, such as joint offsets and link lengths of the manipulator [28]

$\dot{x}_{e}=Y_{k}\left(q_{D}, \dot{q}_{D}\right) \theta_{k}$ 


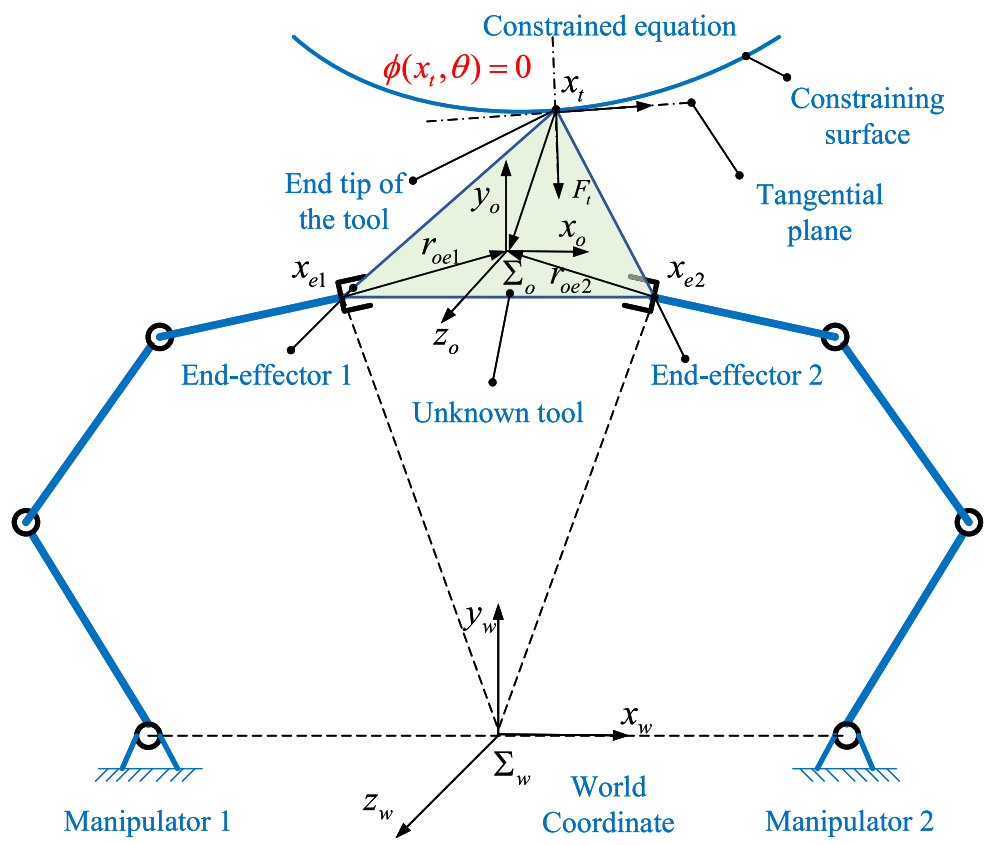

Fig. 1. An example of a dual-arm interconnected system contacting with environment.

where $Y_{k}(.) \in R^{2 m \times j}$ denotes the kinematic regression matrix.

$x_{o} \in R^{p}$ with $p \leq 6$ is defined as the coordinate vector of the object's COM and is assumed that $\dot{x}_{o}$ is related to $\dot{x}_{e}$ by [5]

$\dot{x}_{e}=J_{o} \dot{x}_{o}$

and to the velocity of the object's end tip $\dot{x}_{t} \in R^{p}$ by

$\dot{x}_{o}=\mathcal{R}\left(x_{t}\right) \dot{x}_{t}$

where $J_{o}^{T}=\left[J_{o 1}^{T}, J_{o 1}^{T}\right]^{T} \in R^{p \times 2 m}$ denotes the grasp matrix and $\mathcal{R}\left(x_{t}\right) \in R^{p \times p}$ is assumed invertible. These two matrices can be calculated by $J_{o i}^{T}=\left[I_{3}, 0_{3} ; P_{i}, I_{3}\right]$ for $i=1,2$ and $\mathcal{R}^{T}=$ $\left[I_{3}, O_{3} ; P_{i}, I_{3}\right]$ for $i=t$ (for the case that $p=6$ ), where $P_{i}=S\left(r_{o e i}\right)$ and $r_{o e i}$ denote the vector from object's COM to the corresponding contact point. Definitions of the three components are presented in Fig. 2.

The constraint surface can be defined in an algebraic term as [18]

$\phi\left(x_{t}, \theta\right)=0$

where $\phi\left(x_{t}, \theta\right): R^{p} \rightarrow R^{p-q}$ is a given scalar function and $\theta$ is a known parameter set which depicts the geometry of the constraint surface.

Differentiating Eq. (5) with respect to time yields

$J_{t} \dot{x}_{t}=0$

where $J_{t} \in R^{q \times p}$ denotes the constraint Jacobian of the rigid surface and can be expressed by

$J_{t}=\frac{\partial \phi\left(x_{t}, \theta\right)}{\partial x_{t}}\left\|\frac{\partial \phi\left(x_{t}, \theta\right)}{\partial x_{t}}\right\|^{-1}$ 


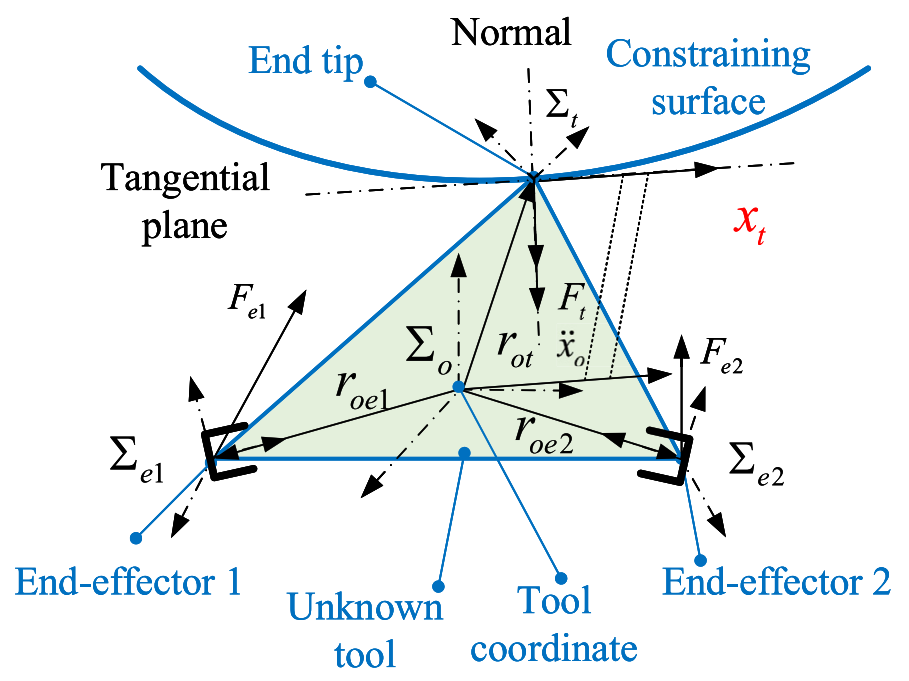

Fig. 2. Sketch of the forces acting on the unknown tool.

\subsubsection{Dynamics}

The dynamic model of the two cooperative manipulators can be expressed in compact form as [29]

$M_{D}\left(q_{D}\right) \ddot{q}_{D}+C_{D}\left(q_{D}, \dot{q}_{D}\right) \dot{q}_{D}+g_{D}\left(q_{D}\right)=\tau-J_{D}^{T} F_{e}$

where $M_{D}\left(q_{D}\right)=\operatorname{blockdiag}\left[M_{1}\left(q_{1}\right), M_{2}\left(q_{2}\right)\right] \in R^{\left(n_{1}+n_{2}\right) \times\left(n_{1}+n_{2}\right)}, M_{i}\left(q_{i}\right) \in R^{n_{i} \times n_{i}}$ denotes the symmetric positive definite inertial matrix of the $i$ th manipulator; $\tau \in R^{\left(n_{1}+n_{2}\right)}$ denotes the applied joint torques and the $F_{e}=\left[\begin{array}{ll}F_{e 1}^{T} & F_{e 1}^{T}\end{array}\right]^{T} \in R^{2 m \times 1}$ is the interacting forces acting on the unknown object; $C_{D} \dot{q}_{D}=\left[\left(C_{1} \dot{q}_{1}\right)^{T},\left(C_{2} \dot{q}_{2}\right)^{T}\right]^{T} \in R^{\left(n_{1}+n_{2}\right) \times 1}$ is the vector of Coriolis and centrifugal forces; $g_{D}=\left[\begin{array}{ll}g_{1}^{T} & g_{2}^{T}\end{array}\right]^{T} \in R^{\left(n_{1}+n_{2}\right) \times 1}$ is the gravitational forces; several essential properties of the manipulator dynamics (8) facilitate the adaptive control design even if this dynamic equation is complex and highly nonlinear [30].

Property 1. $\left(\dot{M}_{D}-2 C_{D}\right)$ is skew-symmetric so that $v^{T}\left(\dot{M}_{D}-2 C_{D}\right) v=0$ for all $v \in R^{n}$.

Property 2. The manipulator dynamics (8) is linear in a set of physical parameters $\theta_{m d r}=$ $\left[\theta_{m d r 1}, \theta_{m d r 2}, \ldots, \theta_{m d r k}\right]^{T} \in R^{k}$.

$M_{D}\left(q_{D}\right) \ddot{q}_{D}+C\left(q_{D}, \dot{q}_{D}\right) \dot{q}_{D}+g_{D}\left(q_{D}\right)=Y_{m d}\left(q_{D}, \dot{q}_{D}, \ddot{q}_{D}\right) \theta_{m d r}$

where the dynamic regression matrix $Y_{m d}(.) \in R^{\left(n_{1}+n_{2}\right) \times k}$ is bounded for bounded argument signals.

The dynamics of the object can be described by the following equation [6]:

$M_{o}\left(x_{o}\right) \ddot{x}_{o}+C_{o}\left(x_{o}, \dot{x}_{o}\right) \dot{x}_{o}+g_{o}\left(x_{o}\right)=F_{o}+\mathcal{R}^{-T} F_{t}$

where $M_{o}\left(x_{o}\right) \in R^{p \times p}$ is the object's inertial matrix; $C_{o}\left(x_{o}, \dot{x}_{o}\right) \dot{x}_{o} \in R^{p}$ denotes the vector of the object's Coriolis and centrifugal forces, and $g_{o}\left(x_{o}\right) \in R^{p}$ represents the vector of gravitational forces; $F_{o} \in R^{p}$ is the resultant force acting on the object's COM by the two manipulators and $F_{t} \in R^{p}$ denotes the contact force exerted by the environment on the end tip of the object. 


\subsection{Force analysis of the dual-arm system}

According to the principle of virtual work, relationship between the resultant force $F_{o}$ and the end-effector force $F_{e}$ can be expressed by

$F_{o}=J_{o}^{T} F_{e}$

Given the force $F_{o}, F_{e}$ can be decomposed into two orthogonal components, one is motioninduced force $F_{E}$ which contributes to the motion of the grasped object, the other one is the internal force $F_{I}$ which contributes to the build-up of the internal strain in the object.

$F_{e}=F_{E}+F_{I}$

where $F_{E}=\left(J_{o}^{T}\left(x_{o}\right)\right)^{\dagger} F_{o}, F_{I}=\mathcal{N} F_{e}$ and $\mathcal{N}=I-\left(J_{o}^{T}\left(x_{o}\right)\right)^{\dagger} J_{o}^{T}\left(x_{o}\right)$ is the null-space matrix of $J_{o}^{T}\left(x_{o}\right)$. For special nonsqueezing pseudoinverse solution of the grasp matrix $J_{o}$, one can refer to [31].

Remark 1. Decomposition of the interaction force is still a controversial research topic in multi-arm cooperation. Walker et al. [31] first proposed a special nonsqueezing pseudoinverse and stated that only one solution can avoid internal loading phenomenon. Chung et al. [32] then challenged this result and used Moore-Penrose inverse instead. Recently, Erhart and Hirche [33] provided a new load distribution from the perspective of kinematic constraint. Based on the results of our previous work, generalized inverse solution indeed cannot achieve nonsqueezing results. Erhart and Hirche only doubted the uniqueness but not correctness of the solution in [31]. So, the solution in [31] is still adopted in this paper.

$F_{I}$ can be parameterized by the vector of Lagrangian multiplier

$F_{I}=\mathcal{F}^{T} \lambda_{I}$

where $\mathcal{F}^{T} \in R^{p}$ is the vector which describes the direction of the internal force and satisfies $J_{o}^{T}\left(x_{o}\right) \mathcal{F}^{T}=0$ and $\mathcal{F} \mathcal{F}^{T}=1, \lambda_{I}$ is the Lagrange multiplier of $F_{I}$.

The contact force between the end tip and constraint surface can also be parameterized as

$F_{t}=J_{t}^{T} \lambda_{t}$

where $\lambda_{t}$ is the Lagrange multiplier of $F_{t}$.

Remark 2. Most of the related papers $[18,34]$ assume that the contact force $F_{t}$ can be measured directly when coping with the force tracking problem. While this assumption does not coincide with the actual situation since the 6-DOF force/torque sensors can only be mounted on the wrists of the robotic arms but not the end tip of the object. Hence estimation of this force is needed.

The force diagram of the object is shown in Fig. 2, from which we can know that projection of the resultant force of the object's gravity $g_{o}\left(x_{o}\right)$ and the applied force $F_{e}$ onto the normal direction of the tangential plane at the contact point is approximately equal and opposite to the contact force. This analysis leads to the following estimation equation of the contact force:

$F_{t}=\left\|J_{t}^{T}\left(J_{t} J_{t}^{T}\right)^{-1} J_{t}\left(F_{e 1}+F_{e 2}+g_{o}\right)\right\| J_{t}^{T}$

where $J_{t}^{T}\left(J_{t} J_{t}^{T}\right)^{-1} J_{t}$ is the projection matrix. 


\section{Synthesis of the control law}

In the presence of uncertainty in the closed kinematic chain, the Jacobian matrix of the dual-arm system and the grasp matrix are uncertain. Then Eq. (2) should be rewritten as

$\hat{\dot{x}}_{e}=Y_{k}\left(q_{D}, \dot{q}_{D}\right) \hat{\theta}_{k}$

where $\hat{\theta}_{k}$ is the estimate of $\theta_{k}$.

Combining Eqs. (3) and (4) when considering the uncertainties yields

$\hat{\dot{x}}_{t}=\hat{\mathcal{R}}^{-} \hat{J}_{o}^{\dagger} \hat{J}_{D} \dot{q}_{D}=\hat{\mathcal{R}}^{-} \hat{J}_{o}^{\dagger} Y_{k} \hat{\theta}_{k}$

where $\hat{J}_{o}$ and $\hat{J}_{D}$ are the estimates of $J_{o}$ and $J_{D}$.

Remark 3. Since the grasp matrix $J_{o}$ not only determines the kinematic relationship between the object space and end-effector space but also determines the decomposition of the applied force $F_{e}$, estimation of $J_{o}$ should be specially implemented and its convergence needs to be always guaranteed even without sufficient persistent excitation condition.

\subsection{Estimation of the object's centre of mass}

Inspired from [22], virtual link concept can be utilized and reformulated to achieve convergent estimation of the object's COM in this context.

$R_{o} r_{o}$ can be linearly parameterized as

$R_{o} r_{o}=R\left(x_{o}\right) r$

where $r_{o}=\left[{ }^{w} r_{o e 1}^{T},{ }^{w} r_{o e 2}^{T},{ }^{w} r_{o t}^{T}\right]^{T}$ is the combined vector, $r_{o i}$ for $i=e 1, e 2, t$ denotes the vector from object's COM to corresponding contact points expressed in the object frame; $R_{o}=$ blockdiag $\left[{ }^{w} R_{o},{ }^{w} R_{o},{ }^{w} R_{o}\right]$ and ${ }^{w} R_{o}$ denotes the rotation matrix from object frame to world frame; $R\left(x_{o}\right)=\operatorname{blockdiag}\left[R_{1}, R_{2}, R_{3}\right]$ is the regression matrix and the $r=\left[r_{1}^{T}, r_{2}^{T}, r_{3}^{T}\right]^{T}$ is the parameter vector. One can obtain clear understanding of these definitions from Fig. 2.

Then estimated position of the object's COM can be updated by the following laws:

$$
\begin{aligned}
\hat{X}_{e t} & =\hat{X}_{o}+R \hat{r} \\
e & =X_{e t}-\hat{X}_{e t} \\
\dot{\hat{r}} & =\varphi R^{T} e-v \hat{r} \\
\hat{x}_{o} & =\frac{1}{3}\left(x_{e 1}+x_{e 2}+x_{t}-\sum_{i=1}^{3} R_{i} \hat{r}_{i}\right)
\end{aligned}
$$

where $X_{e t}=\left[x_{e 1}^{T}, x_{e 2}^{T}, x_{t}^{T}\right]^{T}, X_{o}=\left[x_{o}^{T}, x_{o}^{T}, x_{o}^{T}\right]^{T} ; \varphi$ and $v$ are positive gain constants. This iterative Newton-Raphson method can achieve convergent estimation of the object's COM, and further the grasp/transformation matrix [35]. Its self-convergence and independence from the control scheme enable us to concentrate on the handling of multiple objectives under robot kinematic and system dynamic uncertainties (including robot dynamic and object dynamic uncertainties). Convergence analysis is provided in Appendix A.

\subsection{Design of the adaptive hybrid position/force controller}

First, define the estimation velocity error as

$$
\Delta \hat{\dot{x}}_{t}=\dot{x}_{t d}-\hat{\dot{x}}_{t}
$$


where $\dot{x}_{t d}$ is the desired tip velocity.

Define the contact force tracking error $\Delta \lambda_{t}$ and internal force tracking error $\Delta \lambda_{I}$ as

$\Delta \lambda_{t}=\lambda_{t d}-\lambda_{t}$

$\Delta \lambda_{I}=\lambda_{I d}-\lambda_{I}$

where $\lambda_{t d}$ and $\lambda_{I d}$ are desired contact force and internal force.

The following two force error signals are introduced to avoid the force derivatives in the controller since they are often very noisy:

$\Delta \lambda_{F t}=\int_{0}^{t} \Delta \lambda_{t}(\sigma) d \sigma$
$\Delta \lambda_{F I}=\int_{0}^{t} \Delta \lambda_{I}(\sigma) d \sigma$

Define the reference tip velocity $\dot{x}_{t r}$ as

$\dot{x}_{t r}=\dot{x}_{t d}+\alpha\left(x_{t d}-x_{t}\right)-\beta R_{t} J_{t}^{T} \Delta \lambda_{F t}$

where $x_{t d}$ is desired tip position; $\alpha$ and $\beta$ are positive constants; $R_{t}$ is a rotation matrix which will be defined later.

Considering Eqs. (3) and (4) with internal force control objective, the reference joint velocity is defined as

$$
\begin{aligned}
& \dot{q}_{r}={\hat{J_{D}}}_{D}^{\dagger}\left(J_{o} \mathcal{R} \dot{x}_{t r}+\kappa N_{J_{o}^{\dagger}} \mathcal{F}^{T} \lambda_{F I}\right)+\left(I-\hat{J}_{D}^{\dagger} \hat{J_{D}}\right) \psi \\
& ={\hat{J_{D}}}_{D}^{\dagger} J_{o} \mathcal{R}[\underbrace{\dot{x}_{t d}+\alpha\left(x_{t d}-x_{t}\right)}_{\text {Tip position term }}-\underbrace{\beta R_{t} J_{t}^{T} \Delta \lambda_{F t}}_{\text {Contact force term }}]+\underbrace{\kappa \hat{J}_{D}^{\dagger} N_{J_{o}^{\dagger}} \mathcal{F}^{T} \Delta \lambda_{F I}}_{\text {Internal force term }}+\left(I-\hat{J}_{D}^{\dagger} \hat{J}_{D}\right) \psi
\end{aligned}
$$

where $\hat{J}_{D}^{\dagger}$ is the pseudoinverse of $\hat{J}_{D}$; $I$ is the identity matrix with proper dimensions; $\kappa$ is a positive constant; $\psi$ is minus the gradient of a convex function for optimization; $N_{J_{o}^{\dagger}}$ is the null-space matrix of $J_{o}^{\dagger}$ and satisfy $J_{o}^{\dagger} N_{J_{o}^{\dagger}}=0$.

$$
\begin{aligned}
\hat{J}_{D}^{\dagger} & =\sum_{i=1}^{m_{1}} \frac{\sigma_{i}}{\sigma_{i}^{2}+\lambda_{G i}^{2}} v_{i} \mu_{i}^{T} \\
\lambda_{G i} & =\lambda_{\max } \exp \left(-\left(\sigma_{i} / \varepsilon\right)^{2}\right)
\end{aligned}
$$

in which $\sigma_{i}$ is the singular value of the estimated Jacobian $\hat{J_{D}} ; v_{i}$ and $\mu_{i}$ are the $i$ th output and input singular vectors; $m_{1}$ denotes the number of nonnull singular value of $\hat{J}_{D}$; $\varepsilon$ is the pre-specified constant which defines the size of the singular region, $\lambda_{\max }$ sets the maximum of the damping factor.

Remark 4. A modified singularity robust technique expressed by Eq. (27), first proposed by Ren et al. [36], is adopted here to avoid kinematic singularity. Different from the most commonly used one proposed by Chiaverini [37] in real-time kinematic resolution, this method can ensure continuity and good shaping of $\hat{J}_{D}^{\dagger}$ and $\dot{\hat{J}}_{D}^{\dagger}$. Unnecessary damping for well-conditioned singular vector can almost be eliminated with proper choice of the parameters, which minimizes the reconstruction error as far as possible. 
Differentiating Eq. (26) with respect to time leads to

$$
\begin{aligned}
\ddot{q}_{r}= & \left(I-\hat{J}_{D}^{\dagger} \hat{J}_{D}\right) \dot{\psi}-\frac{d\left(\hat{J}_{D}^{\dagger} \hat{J}_{D}\right)}{d t} \psi+\frac{d\left(\hat{J}_{D}^{\dagger} J_{o} \mathcal{R}\right)}{d t}\left[\dot{x}_{t d}+\alpha\left(x_{t d}-x_{t}\right)-\beta R_{t} J_{t}^{T} \Delta \lambda_{F t}\right] \\
& +\hat{J}_{D}^{\dagger} J_{o} \mathcal{R}\left[\ddot{x}_{t d}+\alpha\left(\dot{x}_{t d}-\dot{x}_{t}\right)-\beta\left(R_{t} J_{t}^{T} \Delta \lambda_{t}+\frac{d\left(R_{t} J_{t}^{T}\right)}{d t} \Delta \lambda_{F t}\right)\right] \\
& +\kappa \frac{d\left(\hat{J}_{D}^{\dagger} N_{J_{o}^{\dagger}} \mathcal{F}^{T}\right)}{d t} \Delta \lambda_{F I}+\kappa \hat{J}_{D}^{\dagger} N_{J_{o}^{\dagger}} \mathcal{F}^{T} \Delta \lambda_{I}
\end{aligned}
$$

Now define a joint-space sliding variable $s$ :

$s=\dot{q}_{r}-\dot{q}_{D}$

whose time derivative can be given as

$\dot{s}=\ddot{q}_{r}-\ddot{q}_{D}$

Then an auxiliary sliding variable $\bar{s}_{o}$ is defined as

$\bar{s}_{o}=J_{o}^{\dagger} J_{D} S$

In view of the object dynamics, the resultant force $F_{o}$ can be computed by

$$
F_{o}=M_{o}\left(x_{o}\right) \ddot{x}_{o}+C_{o}\left(x_{o}, \dot{x}_{o}\right) \dot{x}_{o}+g_{o}\left(x_{o}\right)-\mathcal{R}^{-T} F_{t}
$$

Folding Eqs. (32) and (11) into Eq. (8) yields

$$
\begin{aligned}
\tau= & M_{D}\left(q_{D}\right) \ddot{q}_{D}+C_{D}\left(q_{D}, \dot{q}_{D}\right) \dot{q}_{D}+g_{D}\left(q_{D}\right) \\
& +J_{D}^{T}\left[\left(J_{o}^{T}\right)^{\dagger}\left(M_{o}\left(x_{o}\right) \ddot{x}_{o}+C_{o}\left(x_{o}, \dot{x}_{o}\right) \dot{x}_{o}+g_{o}\left(x_{o}\right)-\mathcal{R}^{-T} F_{t}\right)+\mathcal{F}^{T} \lambda_{I}\right]
\end{aligned}
$$

where $\dot{x}_{o}=J_{o}^{\dagger} J_{D} \dot{q}_{D}$ can be obtained from Eqs. (1) and (3).

Substituting Eqs. (29) and (31) into Eq. (33) leads to

$$
\begin{aligned}
\tau= & -\left(M_{D} \dot{s}+C_{D} s\right)+M_{D} \ddot{q}_{r}+C_{D} \dot{q}_{r}+g_{D}-J_{D}^{T}\left(J_{o}^{T}\right)^{\dagger}\left(M_{o} \dot{\bar{s}}_{o}+C_{o} \bar{s}_{o}\right) \\
& +J_{D}^{T}\left[\left(J_{o}^{T}\right)^{\dagger}\left(M_{o} \ddot{\bar{x}}_{o r}+C_{o} \dot{\bar{x}}_{o r}+g_{o}-\mathcal{R}^{-T} F_{t}\right)+\mathcal{F}^{T} \lambda_{I}\right]
\end{aligned}
$$

where $\dot{\bar{x}}_{o r}=J_{o}^{\dagger} J_{D} \dot{q}_{r}$ is an auxiliary reference object velocity.

According to Property 1, we can obtain

$$
M_{D} \ddot{q}_{r}+C_{D} \dot{q}_{r}+g_{D}=Y_{m d r}\left(q_{D}, \dot{q}_{D}, \dot{q}_{r}, \ddot{q}_{r}\right) \theta_{m d r}
$$

Then considering the definition of $\dot{\bar{x}}_{o r}$, the following equation can be obtained:

$$
\begin{aligned}
& J_{D}^{T}\left(J_{o}^{T}\right)^{\dagger}\left(M_{o} \ddot{\bar{x}}_{o r}+C_{o} \dot{\bar{x}}_{o r}+g_{o}\right) \\
& \quad=J_{D}^{T}\left(J_{o}^{T}\right)^{\dagger}\left[M_{o}\left(J_{o}^{\dagger} J_{D} \ddot{q}_{r}+\frac{d\left(J_{o}^{\dagger} J_{D}\right)}{d t} \dot{q}_{r}\right)+C_{o} J_{o}^{\dagger} J_{D} \dot{q}_{r}+g_{o}\right]
\end{aligned}
$$

The three terms in the square brackets of Eq. (36) can be parameterized as

$$
M_{o}\left(J_{o}^{\dagger} J_{D} \ddot{q}_{r}+\frac{d\left(J_{o}^{\dagger} J_{D}\right)}{d t} \dot{q}_{r}\right)+C_{o} J_{o}^{\dagger} J_{D} \dot{q}_{r}+g_{o}=Y_{o d r}\left(q_{D}, \dot{q}_{D}, \dot{q}_{r}, \ddot{q}_{r}\right) \theta_{o d r}
$$

Then Eq. (36) can be linearly parameterized in a set of parameters $\theta_{J o d}$ :

$$
J_{D}^{T}\left(J_{o}^{T}\right)^{\dagger} Y_{o d r} \theta_{o d r}=Y_{J o d} \theta_{J o d}
$$


The two terms concerning the contact force and internal force in Eq. (34) can also be linearly parameterized as

$J_{D}^{T}\left(J_{o}^{T}\right)^{\dagger} \mathcal{R}^{-T} J_{t}^{T} \lambda_{t}=Y_{f t}\left(q_{D}\right) \theta_{f t} \lambda_{t}$

$J_{D}^{T} \mathcal{F}^{T} \lambda_{I}=Y_{f I}\left(q_{D}\right) \theta_{f I} \lambda_{I}$

The terms concerning $s$ and $\bar{s}_{o}$ in Eq. (34) can be reformulated as

$$
\begin{aligned}
& M_{D} \dot{s}+C_{D} s+J_{D}^{T}\left(J_{o}^{T}\right)^{\dagger}\left(M_{o} \dot{\bar{s}}_{o}+C_{o} \bar{s}_{o}\right) \\
& =\left(M_{D}+J_{D}^{T}\left(J_{o}^{T}\right)^{\dagger} M_{o} J_{o}^{\dagger} J_{D}\right) \dot{s}+\left[C_{D}+J_{D}^{T}\left(J_{o}^{T}\right)^{\dagger}\left(C_{o} J_{o}^{\dagger} J_{D}+M_{o} \frac{d\left(J_{o}^{\dagger} J_{D}\right)}{d t}\right)\right] s \\
& =M_{e} \dot{s}+C_{e} s
\end{aligned}
$$

where

$$
\begin{aligned}
& M_{e}=M_{D}+J_{D}^{T}\left(J_{o}^{T}\right)^{\dagger} M_{o} J_{o}^{\dagger} J_{D} \\
& C_{e}=C_{D}+J_{D}^{T}\left(J_{o}^{T}\right)^{\dagger} C_{o} J_{o}^{\dagger} J_{D}+J_{D}^{T}\left(J_{o}^{T}\right)^{\dagger} M_{o} \frac{d\left(J_{o}^{\dagger} J_{D}\right)}{d t}
\end{aligned}
$$

The matrix $\dot{M}_{e}-2 C_{e}$ is a skew symmetric matrix.

Proof. Please see Appendix B.

Combining Eqs. (34), (35) and (37)-(40) yields

$M_{e} \dot{s}+C_{e} s=Y_{m d r} \theta_{m d r}-\tau+Y_{J o d} \theta_{J o d}-J_{D}^{T}\left(J_{o}^{T}\right)^{\dagger} \mathcal{R}^{-T} J_{t}^{T} \lambda_{t}+J_{D}^{T} \mathcal{F}^{T} \lambda_{I}$

Based on the above error analysis, the following adaptive controller is proposed:

$$
\begin{aligned}
& \tau=K_{p} s+\underbrace{Y_{m d r} \hat{\theta}_{m d r}+Y_{J o d} \hat{\theta}_{J o d}-Y_{f t} \hat{\theta}_{f t} \lambda_{t}+Y_{f I} \hat{\theta}_{f I} \lambda_{I}}_{\text {Dynamic compensation }}+\underbrace{\hat{J}_{D}^{T} \mathcal{F}^{T}\left(\Delta \lambda_{I}+\gamma \Delta \lambda_{F I}\right)}_{\text {Internal force control }} \\
& +\left(\mathcal{R}^{-} J_{o}^{\dagger} \hat{J}_{D}\right)^{T}\{\underbrace{K\left(\Delta \hat{\dot{x}}_{t}+\alpha \tilde{x}_{t}\right)}_{\text {Tip position control }}-\underbrace{R_{t} J_{t}^{T}\left(\Delta \lambda_{t}+\gamma \Delta \lambda_{F t}\right)}_{\text {Contact force control }}\}
\end{aligned}
$$

where $\tilde{x}_{t}=x_{t d}-x_{t}$ is the tracking error of the end tip; $K_{p}$ and $K$ are positive definite gain matrix; $\gamma$ is a positive constant; The parameter estimates $\hat{\theta}_{m d r}, \hat{\theta}_{J o d}, \hat{\theta}_{f t} \hat{\theta}_{f I}$ and $\hat{\theta}_{k}$ are updated by

$\dot{\hat{\theta}}_{m d r}=L_{d} Y_{m d r}^{T} s$

$\dot{\hat{\theta}}_{J o d}=L_{J o d} Y_{J o d}^{T} S$

$\dot{\hat{\theta}}_{f t}=-L_{f t} Y_{f t}^{T} s \lambda_{t}$

$\dot{\hat{\theta}}_{f I}=L_{f I} Y_{f I}^{T} s \lambda_{I}$

$\dot{\hat{\theta}}_{k}=-2 L_{k} Y_{k}^{T}\left(\mathcal{R}^{-} J_{o}^{\dagger}\right)^{T} K\left(\dot{\tilde{x}}_{t}+\alpha \tilde{x}_{t}\right)$

where $L_{d}, L_{J o d}, L_{f t}, L_{f I}$ and $L_{k}$ are positive definite gain matrices with proper dimensions. 


\section{Stability and convergence analysis}

Before establishing the convergence of the proposed controller, we would like to introduce the following lemma first.

Lemma 1. [38]. If $f, \dot{f} \in L_{\infty}$ and $f \in L_{p}$ for some $p \in[1, \infty)$, then $f(t) \rightarrow 0$ as $t \rightarrow \infty$.

Define parameter estimation error as

$\tilde{\theta}_{s u b}=\hat{\theta}_{\text {sub }}-\theta_{\text {sub }}$

where the subscript sub denotes the relevant parameters above.

By incorporating the controller (44) into the system dynamics (43), we can obtain

$$
\begin{aligned}
M_{e} \dot{s}+C_{e} s= & -K_{p} s-Y_{m d r} \tilde{\theta}_{m d r}-Y_{J o d} \tilde{\theta}_{J o d}+Y_{f t} \tilde{\theta}_{f t} \lambda_{t}-Y_{f I} \tilde{\theta}_{f I} \lambda_{I} \\
& -\left(\mathcal{R}^{-} J_{o}^{\dagger} \hat{J}_{D}\right)^{T}\left[K\left(\Delta \hat{\dot{x}}_{t}+\alpha \tilde{x}_{t}\right)-R_{t} J_{t}^{T}\left(\Delta \lambda_{t}+\gamma \Delta \lambda_{F t}\right)\right] \\
& -\hat{J}_{D}^{T} \mathcal{F}^{T}\left(\Delta \lambda_{I}+\gamma \Delta \lambda_{F I}\right)
\end{aligned}
$$

To analyse the stability and convergence of this closed-loop system, a Lyapunov candidate function is given as

$$
\begin{aligned}
V= & \frac{1}{2} s^{T} M_{e} s+\alpha \tilde{x}_{t}^{T} K \tilde{x}_{t}+\frac{1}{2} \tilde{\theta}_{m d r}^{T} L_{d}^{-1} \tilde{\theta}_{m d r}+\frac{1}{2} \tilde{\theta}_{J o d}^{T} L_{J o d}^{-1} \tilde{\theta}_{J o d}+\frac{1}{2} \tilde{\theta}_{k}^{T} L_{k}^{-1} \tilde{\theta}_{k} \\
& +\frac{1}{2} \tilde{\theta}_{f t}^{T} L_{f t}^{-1} \tilde{\theta}_{f t}+\frac{1}{2} \tilde{\theta}_{f I}^{T} L_{f I}^{-1} \tilde{\theta}_{f I}+\frac{1}{2} \beta \Delta \lambda_{F t}^{2}+\frac{1}{2} \kappa \Delta \lambda_{F I}^{2}
\end{aligned}
$$

Differentiating $V$ with respect to time leads to

$$
\begin{aligned}
\dot{V}= & s^{T}\left(M_{e} \dot{s}+\frac{1}{2} \dot{M}_{e} s\right)+2 \alpha \tilde{x}_{t}^{T} K \dot{\tilde{x}}_{t}+\tilde{\theta}_{m d r}^{T} L_{d}^{-1} \dot{\hat{\theta}}_{m d r}+\tilde{\theta}_{J o d}^{T} L_{J o d}^{-1} \dot{\hat{\theta}}_{J o d} \\
& +\tilde{\theta}_{f t}^{T} L_{f t}^{-1} \dot{\hat{\theta}}_{f t}+\tilde{\theta}_{f I}^{T} L_{f I}^{-1} \dot{\hat{\theta}}_{f I}+\tilde{\theta}_{k}^{T} L_{k}^{-1} \dot{\hat{\theta}}_{k}+\beta \Delta \lambda_{F t} \Delta \lambda_{t}+\kappa \Delta \lambda_{F I} \Delta \lambda_{I}
\end{aligned}
$$

Substituting the closed-loop dynamics (51) and the four parameter adaptive laws (45)-(49) into Eq. (53) yields

$$
\begin{aligned}
\dot{V}= & -s^{T} K_{p} s-s^{T}\left(\mathcal{R}^{-} J_{o}^{\dagger} \hat{J}_{D}\right)^{T}\left[K\left(\Delta \hat{\dot{x}}_{t}+\alpha \tilde{x}_{t}\right)-R_{t} J_{t}^{T}\left(\Delta \lambda_{t}+\gamma \Delta \lambda_{F t}\right)\right] \\
& -s^{T} \hat{J}_{D}^{T} \mathcal{F}^{T}\left(\Delta \lambda_{I}+\gamma \Delta \lambda_{F I}\right)+2 \alpha \tilde{x}_{t}^{T} K \dot{\tilde{x}}_{t}+\beta \Delta \lambda_{F t} \Delta \lambda_{t}+\kappa \Delta \lambda_{F I} \Delta \lambda_{I} \\
& -2 \tilde{\theta}_{k}^{T} Y_{k}^{T}\left(\mathcal{R}^{-} J_{o}^{\dagger}\right)^{T} K\left(\dot{\tilde{x}}_{t}+\alpha \tilde{x}_{t}\right) \\
& =\dot{V}_{1}+\dot{V}_{2}+\dot{V}_{3}
\end{aligned}
$$

where 


$$
\left\{\begin{array}{l}
\dot{V}_{1}=-\left(\Delta \hat{\dot{x}}_{t}+\alpha \tilde{x}_{t}\right)^{T} K\left(\Delta \hat{\dot{x}}_{t}+\alpha \tilde{x}_{t}\right)+2 \alpha \tilde{x}^{T} K \dot{\tilde{x}}-2 \tilde{\theta}_{k}^{T} Y_{k}^{T}\left(\mathcal{R}^{-} J_{o}^{\dagger}\right)^{T} K\left(\dot{\tilde{x}}_{t}+\alpha \tilde{x}_{t}\right) \\
-s^{T} K_{p} s \\
\dot{V}_{2}=-\beta\left(R_{t} J_{t}^{T} \Delta \lambda_{F t}\right)^{T}\left[-R_{t} J_{t}^{T}\left(\Delta \lambda_{t}+\gamma \Delta \lambda_{F t}\right)+K\left(\Delta \hat{\dot{x}}_{t}+\alpha \tilde{x}_{t}\right)\right]+\beta \Delta \lambda_{F t} \Delta \lambda_{t} \\
\quad+\left(\Delta \hat{\dot{x}}_{t}+\alpha \tilde{x}_{t}\right)^{T} R_{t} J_{t}^{T}\left(\Delta \lambda_{t}+\gamma \Delta \lambda_{F t}\right) \\
\dot{V}_{3}=-s^{T} \hat{J}_{D}^{T} \mathcal{F}^{T}\left(\Delta \lambda_{I}+\gamma \Delta \lambda_{F I}\right)+\kappa \Delta \lambda_{F I} \Delta \lambda_{I}
\end{array}\right.
$$

According to the definition of $\Delta \hat{\dot{x}}_{t}, \dot{\tilde{x}}_{t}$ and $\tilde{\theta}_{k}$, we can obtain

$$
\begin{aligned}
& \dot{\tilde{x}}_{t}-\Delta \hat{\dot{x}}_{t}=\hat{\dot{x}}_{t}-\dot{x}_{t} \\
& =\mathcal{R}^{-} J_{o}^{\dagger} Y_{k} \tilde{\theta}_{k}
\end{aligned}
$$

Folding the above equation into $\dot{V}_{1}$ leads to

$$
\begin{aligned}
\dot{V}_{1}= & -\left(\dot{\tilde{x}}_{t}-\mathcal{R}^{-} J_{o}^{\dagger} Y_{k} \tilde{\theta}_{k}+\alpha \tilde{x}_{t}\right)^{T} K\left(\dot{\tilde{x}}_{t}-R^{-} J_{o}^{\dagger} Y_{k} \tilde{\theta}_{k}+\alpha \tilde{x}_{t}\right)+2 \alpha \tilde{x}_{t}^{T} K \dot{\tilde{x}}_{t} \\
& -2 \tilde{\theta}_{k}^{T} Y_{k}^{T}\left(\mathcal{R}^{-} J_{o}^{\dagger}\right)^{T} K\left(\dot{\tilde{x}}_{t}+\alpha \tilde{x}_{t}\right)-s^{T} K_{p} s \\
& =-\dot{\tilde{x}}_{t}^{T} K \dot{\tilde{x}}_{t}-\left(\mathcal{R}^{-} J_{o}^{\dagger} Y_{k} \tilde{\theta}_{k}\right)^{T} K\left(\mathcal{R}^{-} J_{o}^{\dagger} Y_{k} \tilde{\theta}_{k}\right)-\alpha^{2} \tilde{x}_{t}^{T} K \tilde{x}_{t}-2 \alpha \dot{\tilde{x}}_{t}^{T} K \tilde{x}_{t} \\
& +2 \alpha\left(\mathcal{R}^{-} J_{o}^{\dagger} Y_{k} \tilde{\theta}_{k}\right)^{T} K \tilde{x}_{t}+2 \dot{\tilde{x}}_{t}^{T} K\left(\mathcal{R}^{-} J_{o}^{\dagger} Y_{k} \tilde{\theta}_{k}\right)+2 \alpha \tilde{x}_{t}^{T} K \dot{\tilde{x}}_{t} \\
& -2 \tilde{\theta}_{k}^{T} Y_{k}^{T}\left(\mathcal{R}^{-} J_{o}^{\dagger}\right)^{T} K\left(\dot{\tilde{x}}_{t}+\alpha \tilde{x}_{t}\right)-s^{T} K_{p} s \\
& =-\dot{\tilde{x}}_{t}^{T} K \dot{\tilde{x}}_{t}-\left(\mathcal{R}^{-} J_{o}^{\dagger} Y_{k} \tilde{\theta}_{k}\right)^{T} K\left(\mathcal{R}^{-} J_{o}^{\dagger} Y_{k} \tilde{\theta}_{k}\right)-\alpha^{2} \tilde{x}_{t}^{T} K \tilde{x}_{t}-s^{T} K_{p} s
\end{aligned}
$$

Before simplifying the sub function $\dot{V}_{2}$, the definition the rotation matrix $R_{t}$ is given as follows:

$\bar{s}_{f t} R_{t} J_{t}^{T}=0$

where

$\bar{s}_{f t}=\left(\Delta \hat{\dot{x}}_{t}+\alpha \tilde{x}_{t}\right)^{T}\left[\left(\Delta \lambda_{t}+\gamma \Delta \lambda_{F t}\right)+\beta K \Delta \lambda_{F t}\right]$

$R_{t}$ is introduced here to project the vector of combined force error to the vector of the tip's trajectory tracking error and achieve decoupled motion and force error dynamics.

Considering that $R_{t}^{T} R_{t}=I$ and $J_{t} J_{t}^{T}=1$, then substituting Eqs. (58) and (59) into $\dot{V}_{2}$ yields

$$
\begin{aligned}
\dot{V}_{2} & =-\beta\left(R_{t} J_{t}^{T} \Delta \lambda_{F t}\right)^{T} R_{t} J_{t}^{T}\left(\Delta \lambda_{t}+\gamma \Delta \lambda_{F t}\right)+\beta \Delta \lambda_{F t} \Delta \lambda_{t} \\
& =-\beta \gamma \Delta \lambda_{F t}^{2}
\end{aligned}
$$

Eq. (17) can be reformulated as follows when $\hat{\mathcal{R}} \rightarrow \mathcal{R}$ and $\hat{J}_{o} \rightarrow J_{o}$ $\hat{J}_{D} \dot{q}_{D}=J_{o} \mathcal{R} \hat{\dot{x}}_{t}$

Folding Eqs. (26), (29) and (61) into $\dot{V}_{3}$ leads to $\dot{V}_{3}=-\left(\mathcal{F} \hat{J}_{D} s\right)^{T}\left(\Delta \lambda_{I}+\gamma \Delta \lambda_{F I}\right)+\kappa \Delta \lambda_{F I} \Delta \lambda_{I}$ 


$$
\begin{aligned}
& =-\left[\mathcal{F}\left(J_{o} \mathcal{R} \dot{x}_{t r}+\kappa N_{J_{o}^{\dagger}} \mathcal{F}^{T} \Delta \lambda_{F I}-J_{o} \mathcal{R} \hat{\dot{x}}_{t}\right)\right]^{T}\left(\Delta \lambda_{I}+\gamma \Delta \lambda_{F I}\right)+\kappa \Delta \lambda_{F I} \Delta \lambda_{I} \\
& =-\kappa \gamma \Delta \lambda_{F I}^{2}
\end{aligned}
$$

whose detailed derivation can be found in Appendix C.

Then substituting Eqs. (57), (60) and (62) into Eq. (54) leads to

$$
\begin{aligned}
\dot{V}= & -\dot{\tilde{x}}_{t}^{T} K \dot{\tilde{x}}_{t}-\left(\mathcal{R}^{-} J_{o}^{\dagger} Y_{k} \tilde{\theta}_{k}\right)^{T} K\left(\mathcal{R}^{-} J_{o}^{\dagger} Y_{k} \tilde{\theta}_{k}\right)-\alpha^{2} \tilde{x}_{t}^{T} K \tilde{x}_{t} \\
& -s^{T} K_{p} s-\beta \gamma \Delta \lambda_{F t}^{2}-\kappa \gamma \Delta \lambda_{F I}^{2} \\
& \leq 0
\end{aligned}
$$

Now we are in the position to state the following theorem.

Theorem 1. The adaptive control law (44) with parameter update laws (45)-(49) can guarantee the stability of control system for dual-arm cooperative manipulators and lead to the concurrent convergence of the tracking errors of end tip's motion, contact force and internal force. That is, $\tilde{x}_{t} \rightarrow 0, \dot{\tilde{x}}_{t} \rightarrow 0$ and in addition $\Delta \lambda_{t} \rightarrow 0, \Delta \lambda_{I} \rightarrow 0$ as $t \rightarrow \infty$.

Proof of theorem 1. $\dot{V} \leq 0$ implies that the Lyapunov candidate defined in Eq. (52) is always bounded and non-increasing, which means $s \in \mathcal{L}_{\infty} \cap \mathcal{L}_{2}, \tilde{x}_{t} \in \mathcal{L}_{\infty} \cap \mathcal{L}_{2}, \Delta \lambda_{F t} \in \mathcal{L}_{\infty} \cap \mathcal{L}_{2}$, $\Delta \lambda_{F I} \in \mathcal{L}_{\infty} \cap \mathcal{L}_{2}, \tilde{\theta}_{k}, \tilde{\theta}_{m d r}, \tilde{\theta}_{J o d}, \tilde{\theta}_{J o d L}, \tilde{\theta}_{f t}, \tilde{\theta}_{f I} \in \mathcal{L}_{\infty}, \mathcal{R}^{-} J_{o}^{\dagger} Y_{k} \tilde{\theta}_{k} \in \mathcal{L}_{2}, \dot{\tilde{x}}_{t} \in \mathcal{L}_{2}$.

Considering that all the desired signals are bounded, $\tilde{x}_{t}=x_{t d}-x_{t} \in \mathcal{L}_{\infty}$ leads to $x_{t} \in \mathcal{L}_{\infty}$, and further $q_{D} \in \mathcal{L}_{\infty}$ which means $\hat{J}\left(q_{D}, \hat{\theta}_{k}\right) \in \mathcal{L}_{\infty}$. With $\Delta \lambda_{F t} \in \mathcal{L}_{\infty}$ and $\Delta \lambda_{F I} \in \mathcal{L}_{\infty}$, we can obtain $\dot{q}_{r} \in \mathcal{L}_{\infty}$ from Eq. (26). Together with $s \in \mathcal{L}_{\infty}, \dot{q}_{D} \in \mathcal{L}_{\infty}$ can be concluded from Eq. (29). Then with Eqs. (1), (3) and (4), we have $\dot{x}_{t} \in \mathcal{L}_{\infty}$ and further $\dot{\tilde{x}}_{t} \in \mathcal{L}_{\infty}$ considering $\dot{x}_{t d} \in \mathcal{L}_{\infty}$.

Conclusion 1. With the previous analysis, $\tilde{x}_{t} \in \mathcal{L}_{\infty} \cap \mathcal{L}_{2}$ and $\dot{\tilde{x}}_{t} \in \mathcal{L}_{\infty}$ holds, $\tilde{x}_{t}=x_{t d}-x_{t} \rightarrow$ 0 is achieved according to Lemma 1 .

Differentiating Eq. (25) with respect to time leads to

$$
\ddot{x}_{t r}=\ddot{x}_{t d}+\alpha\left(\dot{x}_{t d}-\dot{x}_{t}\right)-\beta R_{t} J_{t}^{T} \Delta \lambda_{t}-\beta \frac{d\left(R_{t} J_{t}^{T}\right)}{d t} \Delta \lambda_{F t}
$$

Then $z_{x t r}=\ddot{x}_{t r}+\beta R_{t} J_{t}^{T} \Delta \lambda_{t} \in \mathcal{L}_{\infty}$ holds when $\ddot{x}_{t d} \in \mathcal{L}_{\infty} . \dot{\tilde{x}}_{t} \in \mathcal{L}_{\infty}$ and $\tilde{x}_{t} \in \mathcal{L}_{\infty}$ lead to $\dot{\hat{\theta}}_{k} \in \mathcal{L}_{\infty}$ when considering Eq. (49). This further implies $\dot{\hat{J}}\left(q_{D}, \hat{\theta}_{k}\right) \in \mathcal{L}_{\infty}$. From Eq. (28), the following auxiliary variable is defined:

$$
\begin{aligned}
z_{q r}= & \ddot{q}_{r}+\beta \hat{J}_{D}^{\dagger} J_{o} \mathcal{R} R_{t} J_{t}^{T} \Delta \lambda_{t}-\kappa \hat{J}_{D}^{\dagger} N_{J_{o}^{\dagger}} \mathcal{F}^{T} \Delta \lambda_{I} \\
= & \frac{d\left(\hat{J}_{D}^{\dagger} J_{o} \mathcal{R}\right)}{d t}\left[\dot{x}_{t d}+\alpha\left(x_{t d}-x_{t}\right)-\beta R_{t} J_{t}^{T} \Delta \lambda_{F t}\right] \\
& \left.+\hat{J}_{D}^{\dagger} J_{o} \mathcal{R}\left[\ddot{x}_{t d}+\alpha\left(\dot{x}_{t d}-\dot{x}_{t}\right)-\beta \frac{d\left(R_{t} J_{t}^{T}\right)}{d t} \Delta \lambda_{F t}\right)\right]+\kappa \frac{d\left(\hat{J}_{D}^{\dagger} N_{J_{o}^{\dagger}} \mathcal{F}^{T}\right)}{d t} \Delta \lambda_{F I} \\
& -\frac{d\left(\hat{J}_{D}^{\dagger} \hat{J}_{D}\right)}{d t} \psi+\left(I-\hat{J}_{D}^{\dagger} \hat{J}_{D}\right) \dot{\psi}
\end{aligned}
$$


$z_{q r} \in \mathcal{L}_{\infty}$ since the right side of Eq. (65) are all bounded. Furthermore, we can obtain the following equation from Eq. (35):

$$
\begin{aligned}
Y_{m d r}\left(q_{D}, \dot{q}_{D}, \dot{q}_{r}, \ddot{q}_{r}\right) \tilde{\theta}_{m d r} & =\left(\hat{M}_{D}-M_{D}\right) \ddot{q}_{r}+\left(\hat{C}_{D}-C_{D}\right) \dot{q}_{r}+\left(\hat{g}_{D}-g_{D}\right) \\
& =\left(\hat{M}_{D}-M_{D}\right) \ddot{q}_{r}+Z_{m d r}\left(q_{D}, \dot{q}_{D}, \dot{q}_{r}, \hat{\theta}_{m d r}\right)
\end{aligned}
$$

where $Z_{m d r}\left(q_{D}, \dot{q}_{D}, \dot{q}_{r}, \hat{\theta}_{m d r}\right) \in \mathcal{L}_{\infty}$ holds. Then combining Eqs. (37) and (38) yields

$$
\begin{aligned}
Y_{J o d} \tilde{\theta}_{J o d} & =\hat{J}_{D}^{T} J_{o}^{T \dagger} Y_{o d r} \hat{\theta}_{o d r}-J_{D}^{T} J_{o}^{T \dagger} Y_{o d r} \theta_{o d r} \\
& =\left[\hat{J}_{D}^{T}\left(J_{o}^{T}\right)^{\dagger} \hat{M}_{o} J_{o}^{\dagger} \hat{J}_{D}-J_{D}^{T}\left(J_{o}^{T}\right)^{\dagger} M_{o} J_{o}^{\dagger} J_{D}\right] \ddot{q}_{r}+Z_{J o d}\left(q_{D}, \dot{q}_{D}, \dot{q}_{r}, \hat{\theta}_{J o d}\right)
\end{aligned}
$$

where $Z_{J o d}\left(q_{D}, \dot{q}_{D}, \dot{q}_{r}, \hat{\theta}_{J o d}\right) \in \mathcal{L}_{\infty}$. Folding Eqs. (66) and (67) into Eq. (51) leads to

$$
\begin{aligned}
& M_{e} \dot{s}+\left(C_{e}+K_{p}\right) s+\left(\mathcal{R}^{-} J_{o}^{\dagger} \hat{J}_{D}\right)^{T}\left[K\left(\Delta \hat{\dot{x}}_{t}+\alpha \tilde{x}_{t}\right)-R_{t} J_{t}^{T} \gamma \Delta \lambda_{F t}\right]+\left(\hat{M}_{D}-M_{D}\right) \ddot{q}_{r} \\
& +Z_{m d r}\left(q_{D}, \dot{q}_{D}, \dot{q}_{r}, \hat{\theta}_{m d r}\right)+\left[\hat{J}_{D}^{T}\left(J_{o}^{T}\right)^{\dagger} \hat{M}_{o} J_{o}^{\dagger} \hat{J}_{D}-J_{D}^{T}\left(J_{o}^{T}\right)^{\dagger} M_{o} J_{o}^{\dagger} J_{D}\right] \ddot{q}_{r} \\
& +Z_{J o d}\left(q_{D}, \dot{q}_{D}, \dot{q}_{r}, \hat{\theta}_{J o d}\right)-Y_{f I} \tilde{\theta}_{f I} \lambda_{I d}+Y_{f t} \tilde{\theta}_{f t} \lambda_{t d}+\gamma \hat{J}_{D}^{T} \mathcal{F}^{T} \Delta \lambda_{F I} \\
& =Y_{f I} \tilde{\theta}_{f I} \Delta \lambda_{I}-Y_{f t} \tilde{\theta}_{f t} \Delta \lambda_{t}-\hat{J}_{D}^{T} \mathcal{F}^{T} \Delta \lambda_{I}+\left(\mathcal{R}^{-} J_{o}^{\dagger} \hat{J}_{D}\right)^{T} R_{t} J_{t}^{T} \Delta \lambda_{t}
\end{aligned}
$$

Multiplying both sides of the above equation by $J_{t} \mathcal{R}^{-} J_{o}^{\dagger} J_{D} M_{e}^{-1}$ yields $\Sigma(t)=\varpi(t) \Delta \lambda_{t}+\omega(t) \Delta \lambda_{I}$

where $J_{t} \mathcal{R}^{-} J_{o}^{\dagger} J_{D} M_{e}^{-1} \dot{q}_{D}=0$ is used and

$$
\begin{aligned}
\Sigma(t)= & J_{t} \mathcal{R}^{-} J_{o}^{\dagger} J_{D} M_{e}^{-}\left\{( \hat { M } _ { D } + \hat { J } _ { D } ^ { T } ( J _ { o } ^ { T } ) ^ { \dagger } \hat { M } _ { o } J _ { o } ^ { \dagger } \hat { J } _ { D } ) \left(\ddot{q}_{r}+\beta \hat{J}_{D}^{\dagger} J_{o} \mathcal{R} R_{t} J_{t}^{T} \Delta \lambda_{t}\right.\right. \\
& \left.-\kappa \hat{J}_{D}^{\dagger} N_{J_{o}^{\dagger}} \mathcal{F}^{T} \Delta \lambda_{I}\right)+\left(\mathcal{R}^{-} J_{o}^{\dagger} \hat{J}_{D}\right)^{T}\left[K\left(\Delta \hat{\dot{x}}_{t}+\alpha \tilde{x}_{t}\right)+R_{t} J_{t}^{T} \gamma \Delta \lambda_{F t}\right]+\frac{d\left(J_{t} \mathcal{R}^{-} J_{o}^{\dagger} J_{D}\right)}{d t} \dot{q} \\
& \left.+Z_{m d r}\left(q_{D}, \dot{q}_{D}, \dot{q}_{r}, \hat{\theta}_{m d r}\right)-Z_{J o d L}\left(q_{D}, \dot{q}_{D}, \dot{q}_{r}, \hat{\theta}_{k}\right)+\left(C_{e}+K_{p}\right) s-Y_{f I} \tilde{\theta}_{f I} \lambda_{I d}+Y_{f t} \tilde{\theta}_{f t} \lambda_{t d}\right\}
\end{aligned}
$$

$$
\begin{aligned}
\varpi(t)= & J_{t} \mathcal{R}^{-} J_{o}^{\dagger} J_{D} M_{e}^{-}\left[\left(\hat{M}_{D}+\hat{J}_{D}^{T}\left(J_{o}^{T}\right)^{\dagger} \hat{M}_{o} J_{o}^{\dagger} \hat{J}_{D}\right) \beta \hat{J}_{D}^{\dagger} J_{o} \mathcal{R} R_{t} J_{t}^{T}\right. \\
& \left.-Y_{f t} \tilde{\theta}_{f t}+\left(\mathcal{R}^{-} J_{o}^{\dagger} \hat{J}_{D}\right)^{T} R_{t} J_{t}^{T}\right] \\
= & A_{\varpi}(t) J_{t}^{T} \\
\omega(t)= & -J_{t} \mathcal{R}^{-} J_{o}^{\dagger} J_{D} M_{e}^{-}\left[\left(\hat{M}_{D}+\hat{J}_{D}^{T}\left(J_{o}^{T}\right)^{\dagger} M_{o} J_{o}^{\dagger} J_{D}\right) \kappa \hat{J}_{D}^{\dagger} N_{J_{o}^{\dagger}} \mathcal{F}^{T} \Delta \lambda_{I}\right. \\
& \left.-Y_{f I} \tilde{\theta}_{f I}+\hat{J}_{D}^{T} \mathcal{F}^{T} \Delta \lambda_{I}\right] \\
= & A_{\omega}(t) \mathcal{F}^{T}
\end{aligned}
$$

From the above analysis, $\Sigma(t) \in \mathcal{L}_{\infty}, \varpi(t) \in \mathcal{L}_{\infty}$ and $\omega(t) \in \mathcal{L}_{\infty}$ hold, furthermore $\dot{\varpi} \in$ $\mathcal{L}_{\infty}, \dot{\omega} \in \mathcal{L}_{\infty}$ also hold. Multiplying both sides of Eq. (69) by $\mathcal{A}_{t}^{T}$ (who is bounded and 


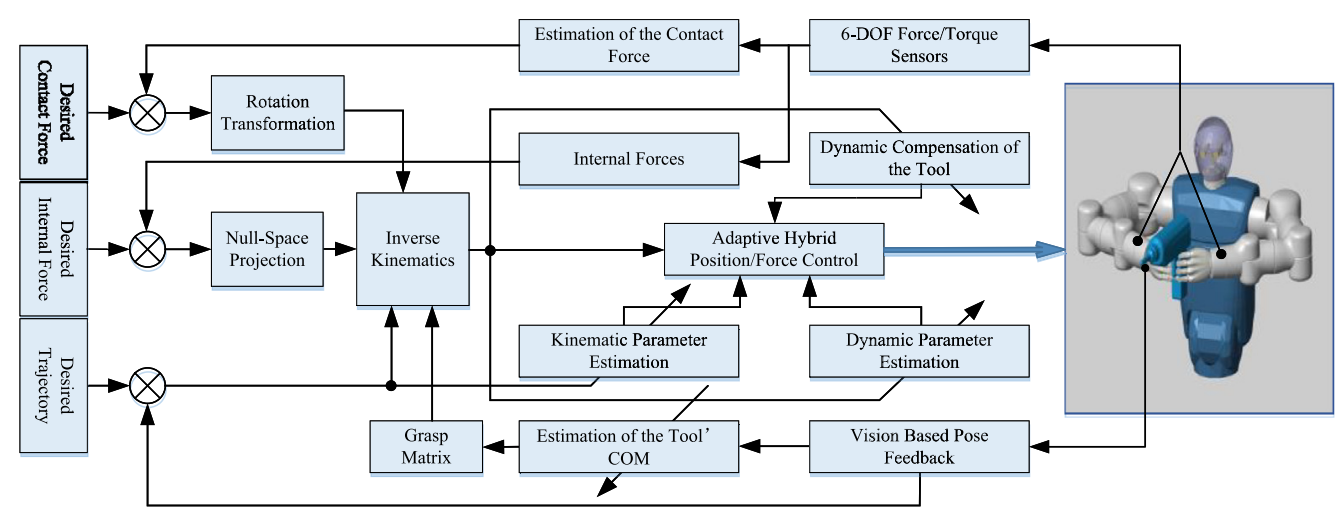

Fig. 3. Block diagram of the adaptive hybrid position/force control architecture of dual-arm redundant manipulators.

satisfies $J_{t} \mathcal{A}_{t}=0$ ) yields $\mathcal{A}_{t}^{T} A_{\varpi}^{\dagger} \Sigma(t)=\mathcal{A}_{t}^{T} A_{\varpi}^{\dagger} A_{\omega} \mathcal{F}^{T} \Delta \lambda_{I}$, which implies $\Delta \lambda_{I} \in \mathcal{L}_{\infty}$ and then $\Sigma(t)-\varpi(t) \Delta \lambda_{t} \in \mathcal{L}_{\infty}, \Delta \lambda_{t} \in \mathcal{L}_{\infty}$ can also be obtained with similar argument. With the fact that $\Delta \lambda_{F t} \in \mathcal{L}_{\infty} \cap \mathcal{L}_{2}$ and $\Delta \lambda_{F I} \in \mathcal{L}_{\infty} \cap \mathcal{L}_{2}$, we have $\Delta \lambda_{F t} \rightarrow 0$ and $\Delta \lambda_{F t} \rightarrow 0$ by using Lemma 1.

From Eqs. (45)-(49), we can obtain $\dot{\hat{\theta}}_{m d r}, \dot{\hat{\theta}}_{J o d}, \dot{\hat{\theta}}_{f t}, \dot{\hat{\theta}}_{f I}, \dot{\hat{\theta}}_{k} \in \mathcal{L}_{\infty}$, then $\dot{z}_{x t} \in \mathcal{L}_{\infty}$ under the assumption that $\dddot{x}_{t d} \in \mathcal{L}_{\infty}$. Then from Eq. (65), $\frac{d z_{q r}}{d t} \in \mathcal{L}_{\infty}$ can be obtained, which further leads to $\Delta \dot{\lambda}_{t} \in \mathcal{L}_{\infty}$ and $\Delta \dot{\lambda}_{I} \in \mathcal{L}_{\infty}$ when considering Eq. (68). Combining the fact $\Delta \lambda_{F t} \rightarrow 0$ and $\Delta \lambda_{F t} \rightarrow 0, \Delta \lambda_{t} \rightarrow 0$ and $\Delta \lambda_{I} \rightarrow 0$ hold according to Barbalat lemma. Besides, from Eqs. (64) and (65), $\ddot{x}_{r} \in \mathcal{L}_{\infty}$ and $\ddot{q}_{r} \in \mathcal{L}_{\infty}$ can be obtained with above conclusions. Using Eq. (68) further leads to $\dot{s} \in \mathcal{L}_{\infty}, \ddot{q}_{D} \in \mathcal{L}_{\infty}$ and $\ddot{x}_{t} \in \mathcal{L}_{\infty}$, which means $\ddot{\tilde{x}}_{t} \in \mathcal{L}_{\infty}$ holds. Then $\dot{\tilde{x}}_{t} \rightarrow 0$ can be obtained by using Barbalat lemma.

\section{Simulation results}

In this section, we present the simulation results of a two identical three-DOF planar manipulators grasping an unknown welding pistol to verify the performance of the adaptive hybrid position/force controller proposed above (AHPF Scheme for short). Three sets of simulations are carried out to show three different situations: free-space motion tracking, position/force tracking with flat constraint surface and that with curved surface. These simulations are implemented by Simulink and SimMechanics 2G. The block diagram is shown in Fig. 3. To show the superiority, a conventional visual servoing controller without adaptation (abbreviated as CHPF scheme hereinafter) is introduced for comparison. Common parameters of the two comparative schemes share same values. For the complete simulation animation, one can download it from http://ees.elsevier.com/fi/download.aspx?id=158792\&guid= 993deb64-96a7-4e6a-96de-6190e01f3697\&scheme $=1$.

We assume that poses of the end tip and the two end-effectors can be obtained from the vision system. Physical parameters of the system are listed in Appendix D. $I_{i}(i=1,2,3, e)$ denotes the moment of inertial. $l_{i}$ and $l_{c i}$ express their link length and the centres of mass. The parameters depicting the position of the object's COM are set as $l_{o 1}=l_{o 2}=$ $l_{o 3}=0.81 \sqrt{3}-0.505 \mathrm{~m}, a=\pi / 6, b=\pi / 6$ and $c=0$, where $r_{o e 1}=\left[l_{o 1} \cos (a) ; l_{o 1} \sin (a)\right]$, $r_{o e 2}=\left[l_{o 2} \cos (b) ; l_{o 2} \sin (b)\right], r_{o e 3}=\left[l_{o 3} \cos (c) ; l_{o 3} \sin (c)\right]$. The two manipulators share same 
parameters. Considering that the inertial matrix of the object $M_{o}$ is diagonal matrix and $C_{o}=0$ when the object is constrained to planar movement in the simulations, linear parameterization (36) can be greatly simplified. Parameterized linearization of the dynamics and kinematics are not listed here for the sake of brevity.

The rotation matrix $R_{t}$ can be calculated by Rodrigues formula. Stiffness of the environment is set as $K_{t}=30000 \mathrm{~N} / \mathrm{m}$. The initial configuration of the 3-DOF manipulator is set as $q_{D}=$ $[\pi / 3, \pi / 2,0,-\pi / 3,-\pi / 2,0,]^{T}$ and the initial value of the end tip position can then be computed as $[0,0.81 \sqrt{3}-0.505,0]^{T}$.

The control parameters of the proposed controller are chosen as:

$\varphi=1000, v=5 ; K_{p}=20 I_{6}, \alpha=20, \gamma=15, K=30 I_{3}, \beta=0.001, \kappa=0.0001, L_{k}=0.2$, $L_{m d r}=3, \hat{\theta}_{m d r}(0)=0.8 \theta_{m d r}, L_{f t}=0.5, L_{f I}=1.2, L_{J o d}=10, \hat{\theta}_{J o d}(0)=0.8 \theta_{J o d}, \hat{\theta}_{k}(0)=$ $0.8 \times[0.4,0.285,0.22,0.4,0.285,0.22]^{T}$. The two parameters of the Gaussian-based DLS method are chosen as $\lambda_{\max }=0.1, \varepsilon=0.05$. The desired internal force and the contact force are set as $\lambda_{I d}=20 \mathrm{~N}$ and $\lambda_{t d}=15 \mathrm{~N}$.

\subsection{Free-space motion tracking}

This simulation is conducted to show the effectiveness of the proposed AHPF in the position and internal force tracking. The desired trajectory of the end tip is given as

$$
\left\{\begin{array}{l}
x_{t d}=0.05+0.1 \times \sin (0.8 \pi t) \\
y_{t d}=0.81 \sqrt{3}-0.505-0.1(1-\cos (0.8 \pi t))+0.06 \\
\theta_{t d}=-t / 60
\end{array}\right.
$$

Simulation results are presented in Fig. 4 from which the following remarks can be concluded:

(1) Compared to the conventional scheme, AHPF scheme can achieve higher convergence rate and smaller tracking error when kinematic and dynamic uncertainties exist (shown by Fig. 4(a)-(d)).

(2) Conventional scheme loses control of the internal forces when the grasp matrix and kinematics of the manipulator are uncertain. While the proposed AHPF can achieve this control objective (shown by Fig. 4(e) and (f)).

(3) Convergent estimation of the object's COM is achieved. The estimation algorithm is compatible with and independent from the adaptive controller. This do contribute to the decouple of multiple kinematic uncertainties and the simplification of the controller design (shown by Fig. 4(g) and (h)).

\subsection{Position/force tracking with flat constraint surface}

Snapshot of this simulation is shown in Fig. 5. The desired trajectory for the end tip is set as

$\left\{\begin{array}{l}x_{t d}=0.08 \cos (0.2 \pi t)-0.08 \\ y_{t d}=0.81 \sqrt{3}-0.505+0.04(1-\cos (0.2 \pi t))+0.03 \\ \theta_{t d}=-t / 60\end{array}\right.$

Constraint equation of the contact surface is depicted by $x_{t} / 2+y_{t}-0.81 \sqrt{3}+0.505-$ $0.03=0$, from which the constraint Jacobian $J t=[-0.5,-1,0]$ can be obtained and the 


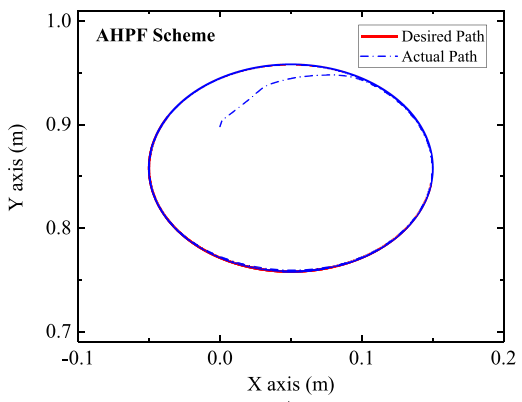

(a)

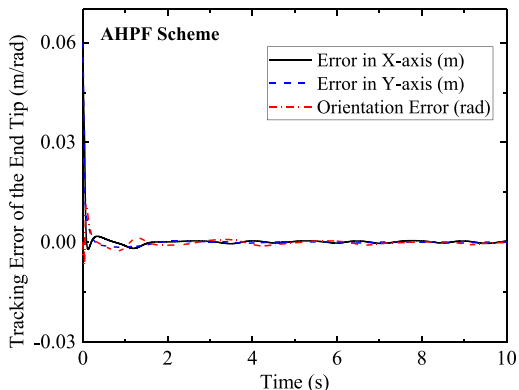

(c)

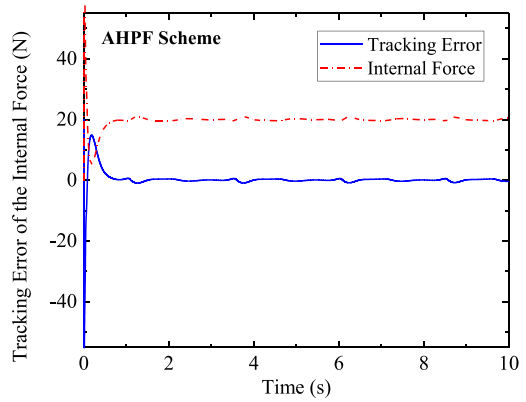

(e)

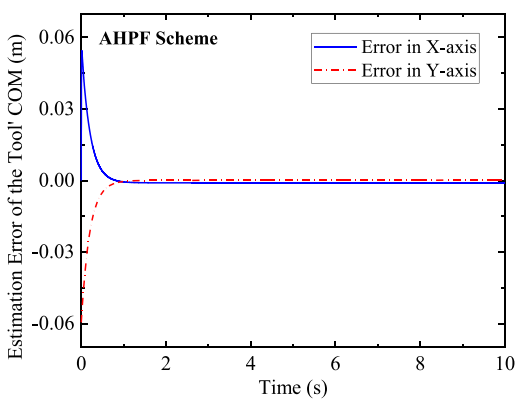

(g)

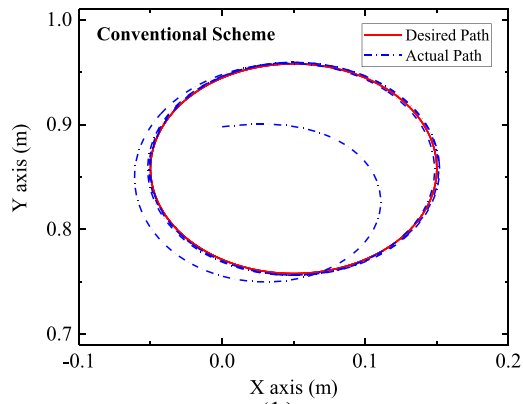

(b)

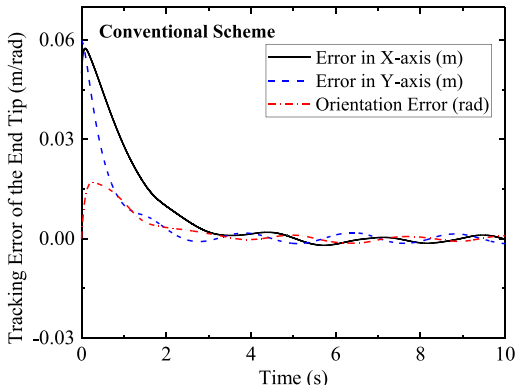

(d)

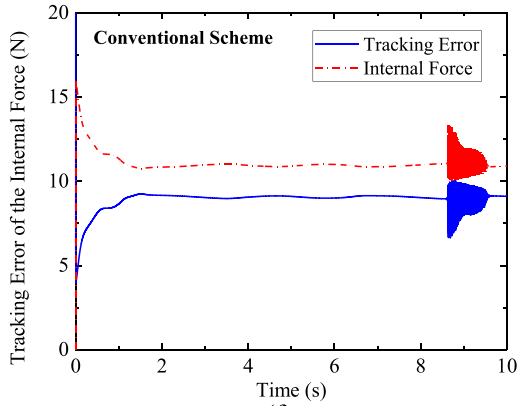

(f)

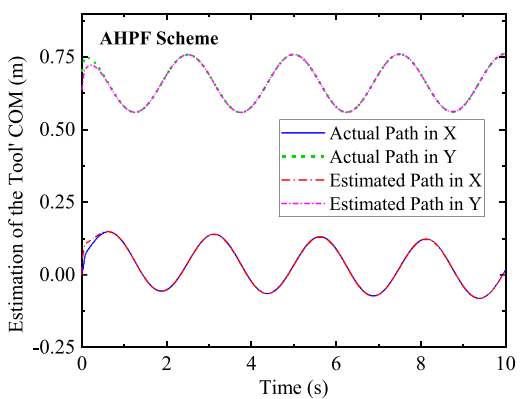

(h)

Fig. 4. Tracking results with two comparative schemes under free-space motion. 


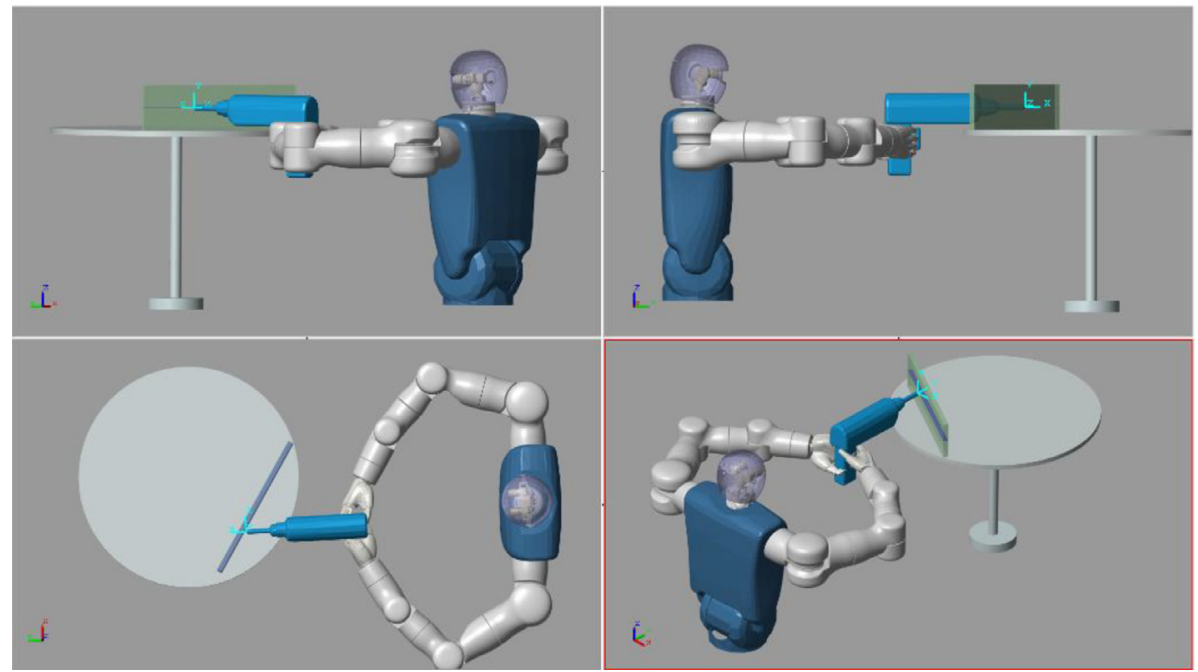

Fig. 5. Snapshot of the flat contact simulation.

contact force is modelled as

$F_{t}=\left\{\begin{array}{l}\frac{2}{\sqrt{5}} K_{t}\left(x_{t} / 2+y_{t}-0.81 \sqrt{3}+0.502\right) J_{t}^{T} \Delta d \geq 0 \\ 0 \Delta d<0\end{array}\right.$

where $\Delta d$ denotes the penetration distance. Here we adopt the most commonly used penalty function method to model the contact force [39]. From the simulation results presented in Figs. 6 and 7, we can conclude that

(1) Compared to CHPF, the proposed AHPF can still maintain high position tracking performance even when the end tip contact with environment (shown in Fig. 6(a)-(d)).

(2) CHPF scheme loses control of the internal force and the contact force tracking error is nonnegligible when uncertainties exist in the closed kinematic chain. While the AHPF scheme can simultaneously achieve these two objectives with small tracking errors (shown by Fig. 7(e)-(h)).

\subsection{Position/force tracking with curved constraint surface}

The desired trajectory for the end tip is set as the following equation and the snapshot of this simulation is presented in Fig. 8:

$\left\{\begin{array}{l}x_{t d}=-0.4 \sin (0.12 \pi \sin (0.2 \pi t)) \\ y_{t d}=0.81 \sqrt{3}-0.505+0.4(1-\cos (0.12 \pi \sin (0.2 \pi t)))+0.03 \\ \theta_{t d}=-t / 50\end{array}\right.$

The constraint surface is a cylinder surface which is depicted by $x_{t}^{2}+$ $\left(y_{t}-0.81 \sqrt{3}+0.075\right)^{2}=0.4^{2}$. Then the constraint Jacobian can be expressed as $J t=\left[x_{t}, y_{t}-0.81 \sqrt{3}+0.075,0\right] / \sqrt{\left(x_{t}^{2}+\left(y_{t}-0.81 \sqrt{3}+0.075\right)^{2}\right)}$. The contact force is 


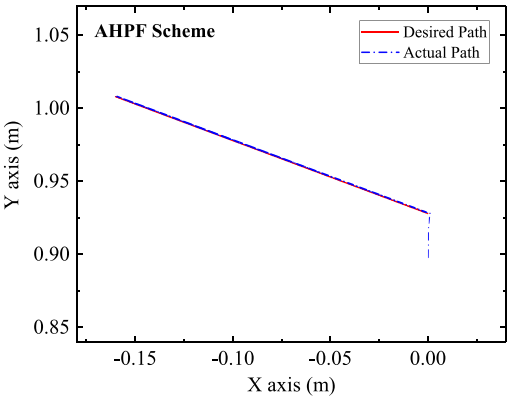

(a)

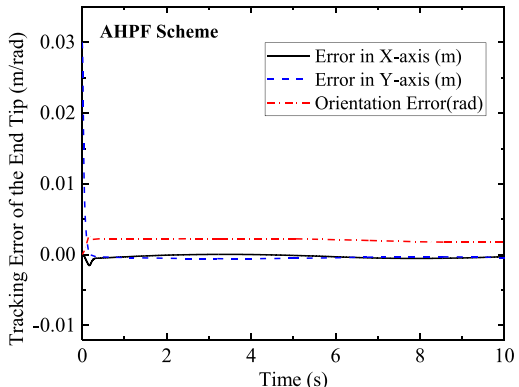

(c)

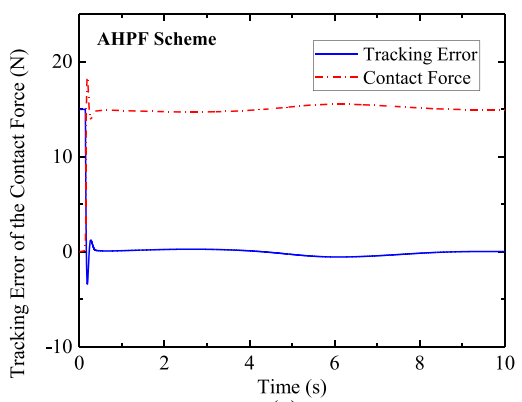

(e)

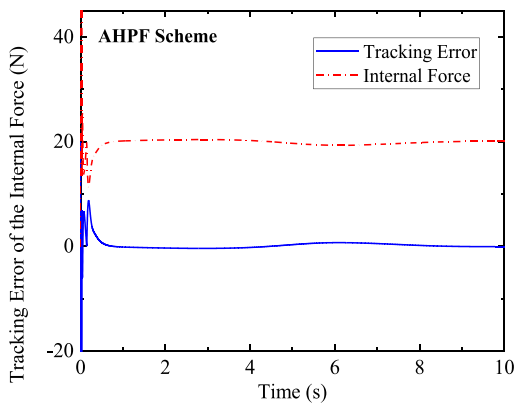

(g)

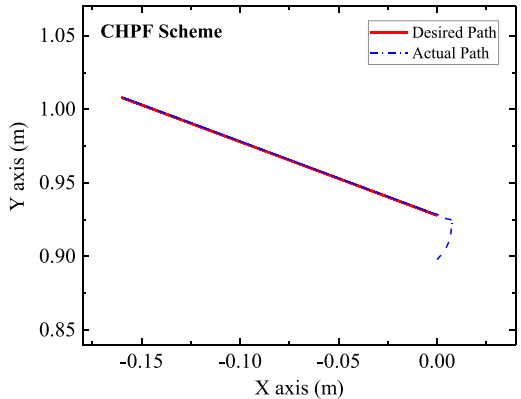

(b)

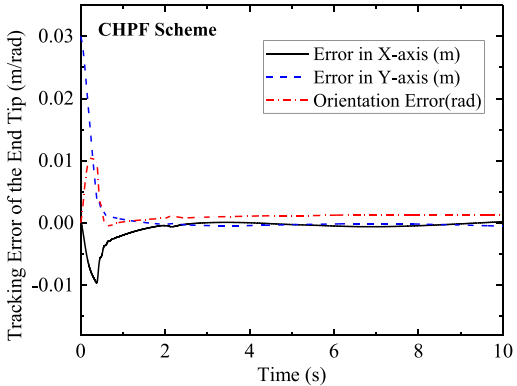

(d)

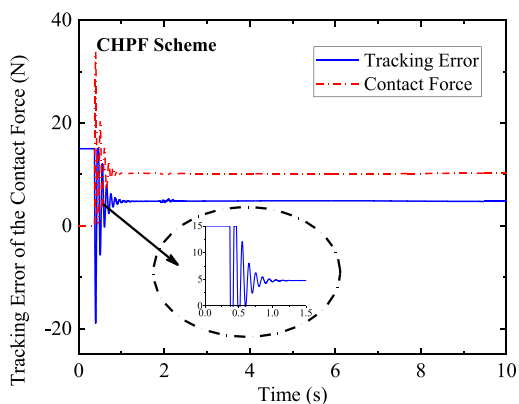

(f)

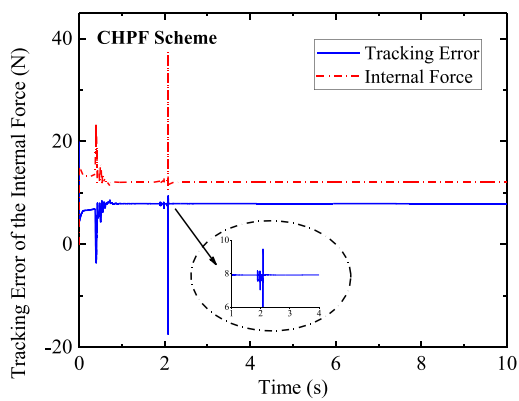

(h)

Fig. 6. Tracking results with two comparative schemes under flat contact. 


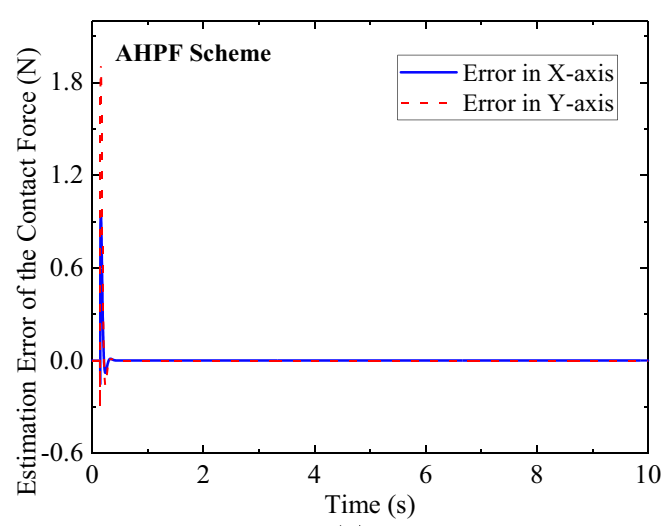

(a)

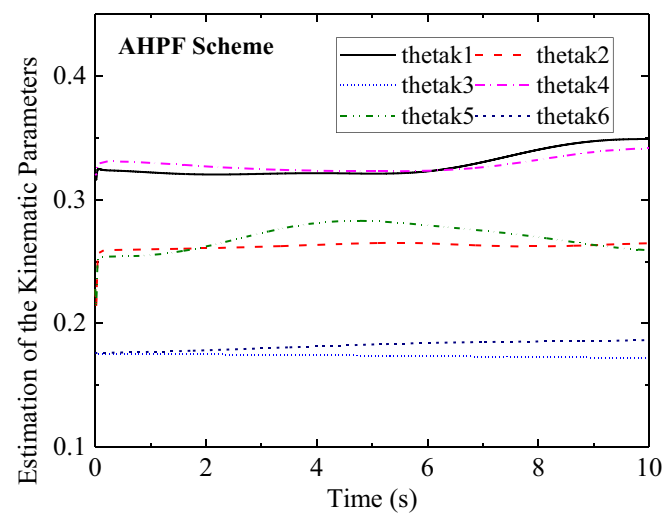

(c)

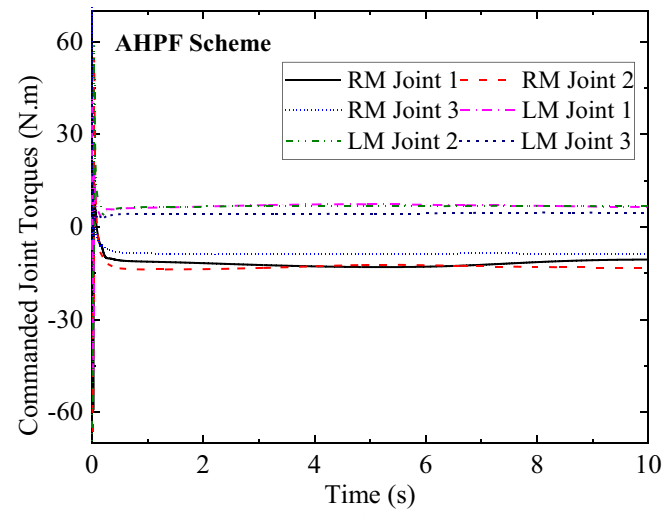

(b)

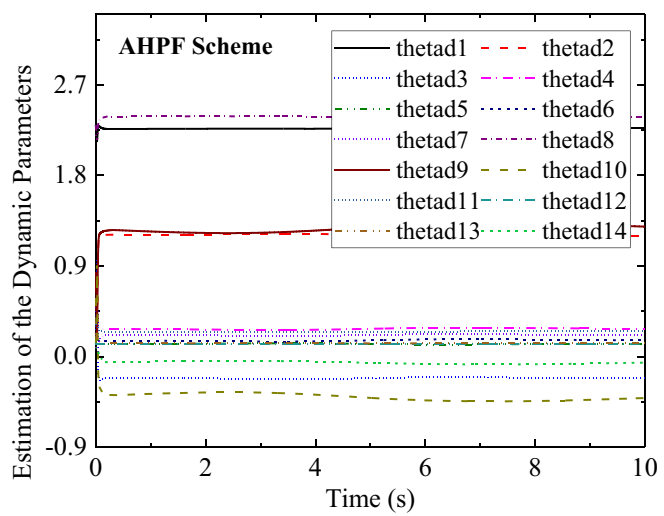

(d)

Fig. 7. Parameter estimation of the flat contact control under AHPF.

modelled as

$F_{t}= \begin{cases}K_{t}\left(0.4-\sqrt{x_{t}^{2}+\left(y_{t}-0.81 \sqrt{3}+0.075\right)^{2}}\right) J_{t}^{T} & \Delta d_{c} \leq 0.4 \\ 0 & \Delta d_{c}>0.4\end{cases}$

where $\Delta d_{c}$ is the distance between the centre of the surface and the end tip. The simulation results are shown in Fig. 9, from which we can see that AHPF can also achieve the concurrent convergence of the end tip's motion error, the contact force error and the internal force error when the object contacts with curved surface.

\section{Conclusion}

In this paper, an adaptive hybrid position/force control strategy has been presented systematically to control dual-arm cooperative manipulators interacting with a rigid surface in the presence of dynamic and close-chain kinematic uncertainties. A practical estimation of the contact force is proposed to implement the force tracking. This adaptive controller is mathematically derived based on Lyapunov stability analysis. No force derivative is needed 


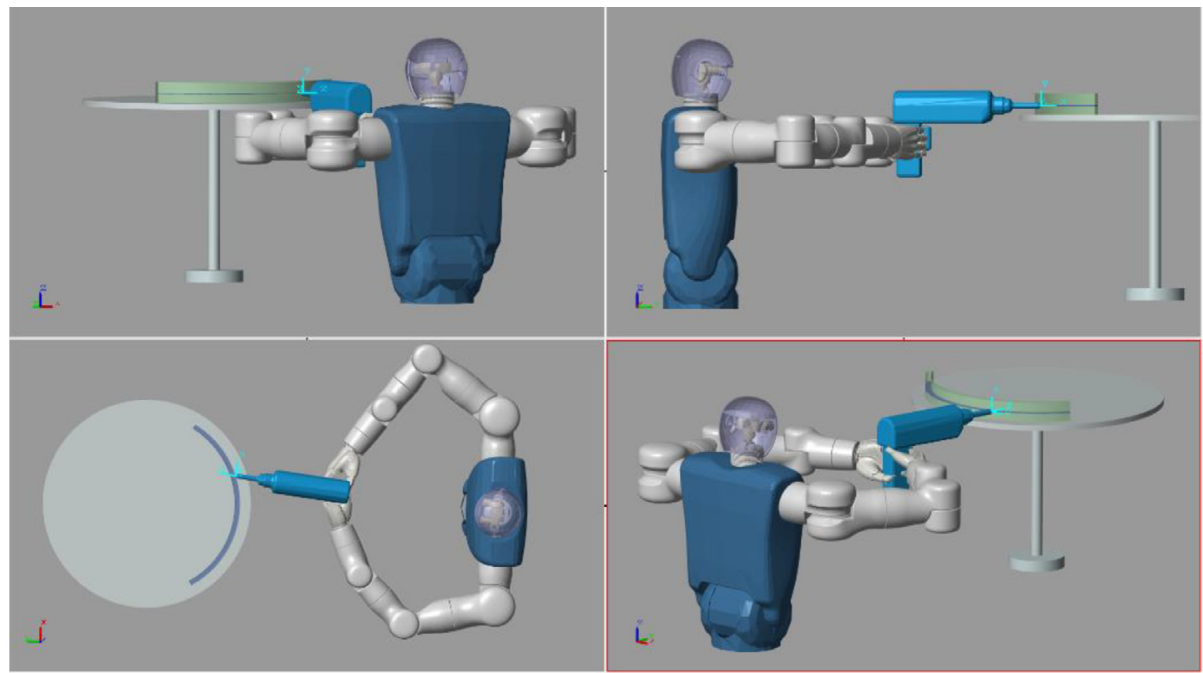

Fig. 8. Snapshot of the curved contact simulation.

and unbiased identification of the grasp matrix is incorporated into the controller by selfconvergent estimation law of the object's COM. Asymptotic convergence of the tracking errors of end tip's motion, contact force and internal force has been achieved. Simulation results have verified the effectiveness and superiority of the proposed controller compared to conventional visual servoing hybrid position/force control scheme.

\section{Acknowledgements}

The authors would like to express their great appreciation to the anonymous reviewers for their precious time. Special thanks to Dr. Hanlei Wang for his suggestions and Dr. Yang Liu for his great help. This work is supported by the Foundation for Innovative Research Groups of the National Natural Science Foundation of China (nos. 51521003 and 61603112).

\section{Appendix A}

Define a cost function

$V_{o}=\frac{1}{2} e^{T} e$

According to Newton-Raphson method, $V_{o}$ and then $e$ can be minimized when the parameter $\hat{r}$ is updated by $\dot{\hat{r}}=-\varphi \frac{\partial V_{o}}{\partial \hat{r}}$ in which $\varphi$ is a constant positive gain. Considering Eq. (19), one can obtain that

$$
\begin{aligned}
\dot{\hat{r}}= & -\varphi \frac{\partial V_{o}}{\partial \hat{r}}=-\varphi \frac{\partial\left(e^{T} e\right)}{2 \partial \hat{r}} \\
& =-\varphi \frac{\partial e^{T}}{\partial \hat{r}} e=-\varphi \frac{\partial\left(X_{e t}-\hat{X}_{o}-R \hat{r}\right)^{T}}{\partial \hat{r}} e \\
& =\varphi R^{T} e
\end{aligned}
$$




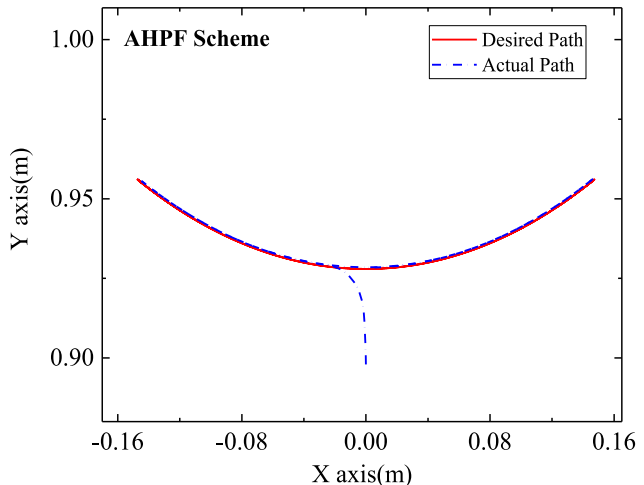

(a)

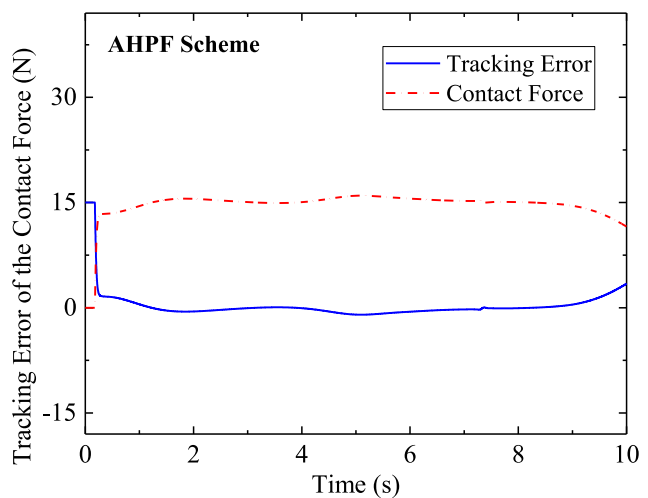

(c)

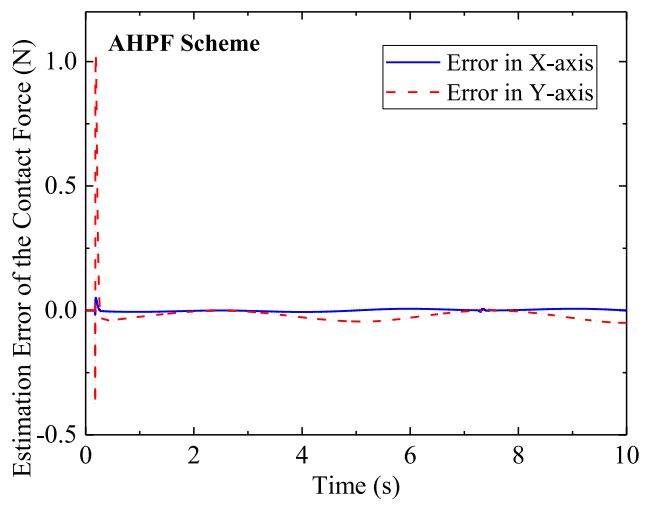

(e)

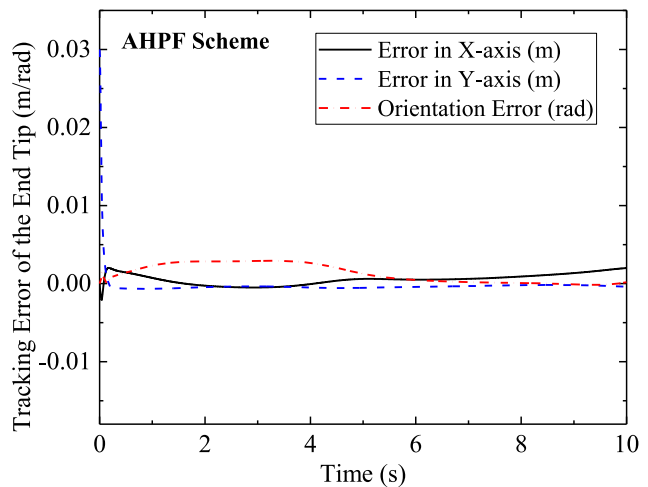

(b)

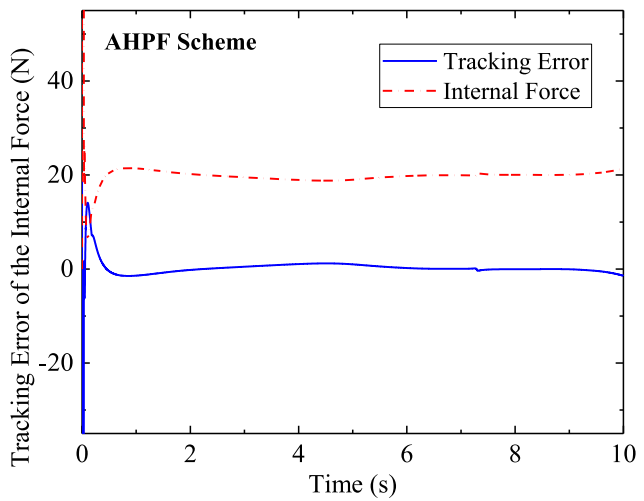

(d)

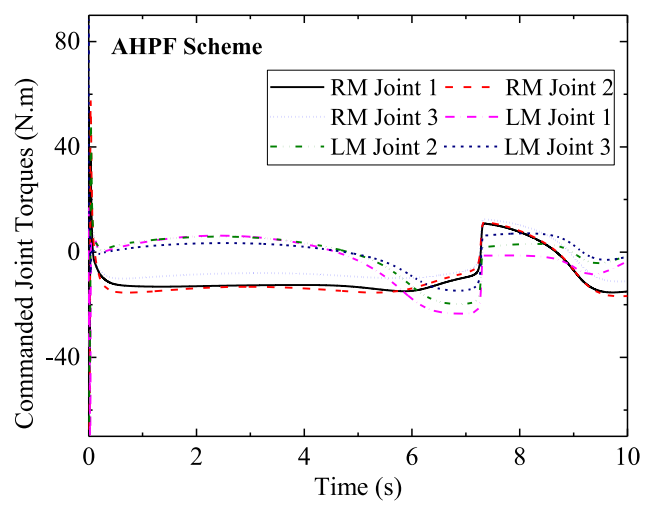

(f)

Fig. 9. The position and force tracking results with AHPF under curved contact.

The damping term in the updating law in Eq. (19) is added to increase the convergence rate of the estimation error. 


\section{Appendix B}

$$
\begin{aligned}
\dot{M}_{e}-2 C_{e}= & \dot{M}_{D}-2 C_{D}+\frac{d\left[J_{D}^{T}\left(J_{o}^{T}\right)^{\dagger} M_{o} J_{o}^{\dagger} J_{D}\right]}{d t} \\
& -2\left[J_{D}^{T}\left(J_{o}^{T}\right)^{\dagger} C_{o} J_{o}^{\dagger} J_{D}+J_{D}^{T}\left(J_{o}^{T}\right)^{\dagger} M_{o} \frac{d\left(J_{o}^{\dagger} J_{D}\right)}{d t}\right] \\
& =\left(\dot{M}_{D}-2 C_{D}\right)+J_{D}^{T}\left(J_{o}^{T}\right)^{\dagger}\left(\dot{M}_{o}-2 C_{o}\right) J_{o}^{\dagger} J_{D}
\end{aligned}
$$

For any $v \in R^{2 n \times 1}$, the following equation always holds:

$$
\begin{aligned}
v^{T}\left(\dot{M}_{e}-2 C_{e}\right) v= & v^{T}\left(\dot{M}_{D}-2 C_{D}\right) v+v^{T} J_{D}^{T}\left(J_{o}^{T}\right)^{\dagger}\left(\dot{M}_{o}-2 C_{o}\right) J_{o}^{\dagger} J_{D} v \\
& =\left(J_{o}^{\dagger} J_{D} v\right)^{T}\left(\dot{M}_{o}-2 C_{o}\right)\left(J_{o}^{\dagger} J_{D} v\right) \\
& =0
\end{aligned}
$$

Therefore, that $\dot{M}_{e}-2 C_{e}$ is skew symmetric is proved.

\section{Appendix C}

$$
\dot{V}_{3}=-\left[\mathcal{F}\left(J_{o} \mathcal{R} \dot{x}_{t r}+\kappa N_{J_{o}^{\dagger}} \mathcal{F}^{T} \Delta \lambda_{F I}-J_{o} R \hat{\dot{x}}_{t}\right)\right]^{T}\left(\Delta \lambda_{I}+\gamma \Delta \lambda_{F I}\right)+\kappa \Delta \lambda_{F I} \Delta \lambda_{I}
$$

can be further simplified by considering $J_{o}^{T}\left(x_{o}\right) \mathcal{F}^{T}=0$

$$
\dot{V}_{3}=-\kappa\left(\mathcal{F} N_{J_{o}^{\dagger}} \mathcal{F}^{T}\right)^{T} \Delta \lambda_{F I}\left(\Delta \lambda_{I}+\gamma \Delta \lambda_{F I}\right)+\kappa \Delta \lambda_{F I} \Delta \lambda_{I}
$$

The null-space matrix $N_{J_{o}^{\dagger}}$ and $N_{J_{o}^{T}}$ satisfy the following equations:

$$
\begin{aligned}
N_{J_{o}^{\dagger}} & =I-J_{o}\left(J_{o}^{T} J_{o}\right)^{-1} J_{o}^{T} \\
N_{J_{o}^{T}} & =N_{J_{o}^{\dagger}} \\
N_{J_{o}^{T}}^{T} & =N_{J_{o}^{T}} \\
N_{J_{o}^{T}} N_{J_{o}^{T}} & =I
\end{aligned}
$$

Considering the above properties, the following reformulation can be obtained:

$$
\begin{aligned}
\mathcal{F} N_{J_{o}^{\dagger}} \mathcal{F}^{T} & =\mathcal{F} N_{J_{o}^{T}} \mathcal{F}^{T}=\left(\left(N_{J_{o}^{T}}\right)^{T} \mathcal{F}^{T}\right)^{T} \mathcal{F}^{T} \\
& =\left(\left(N_{J_{o}^{T}}\right)^{T} \frac{N_{J_{o}^{T}} F_{e}}{\lambda_{I}}\right)^{T} \mathcal{F}^{T}=\mathcal{F} \mathcal{F}^{T} \\
& =1
\end{aligned}
$$

Finally, folding Eq. (C4) into Eq. (C2) yields $\dot{V}_{3}=-\kappa \gamma \Delta \lambda_{F I}^{2}$. 


\section{Appendix D.}

\section{Notations}

\begin{tabular}{|c|c|}
\hline Symbol & Definition \\
\hline$\hat{\mathrm{A}}$ & Estimated form of the related matrix/vector $\mathrm{A}$ \\
\hline$\dot{\mathrm{A}}$ & First-order derivative of the related matrix/vector A \\
\hline$\ddot{\mathrm{A}}$ & Second-order derivative of the related matrix/vector A \\
\hline$\tilde{\mathrm{A}}$ & Parameter estimation error of the related matrix/vector A \\
\hline $\mathrm{A}^{\dagger}$ & Pseudoinverse of the related matrix/vector $\mathrm{A}$ \\
\hline$x_{e}$ & Pose vector of the two end-effectors \\
\hline$q_{D}$ & Joint angle vector of the dual-arm manipulators \\
\hline$J_{D}$ & Jacobian matrix of the dual-arm manipulators ensemble \\
\hline$\theta_{k}$ & Linearized kinematic parameters of the dual-arm manipulators \\
\hline$Y_{k}()$. & Kinematic regression matrix of the dual-arm manipulators \\
\hline$x_{o}$ & Coordinate vector of the object's centre of mass \\
\hline$x_{t}$ & Coordinate vector of the object's end tip \\
\hline$J_{o}$ & Grasp matrix \\
\hline $\mathcal{R}\left(x_{t}\right)$ & Mapping matrix from the task space to object space \\
\hline$r_{o e i}$ & Vector from COM of the object to the corresponding contact point \\
\hline$J_{t}$ & Constraint Jacobian of the rigid surface \\
\hline$\phi\left(x_{t}, \theta\right)$ & Manifold to depict the constraint surface \\
\hline$\theta$ & Constant parameter to depict the constraint surface \\
\hline$M_{D}\left(q_{D}\right)$ & Inertial matrix of the dual-arm manipulators \\
\hline$\tau$ & Applied joint torques of the dual-arm manipulators \\
\hline$F_{e}$ & Interacting forces exerted by the two end-effectors on the object \\
\hline$C_{D} \dot{q}_{D}$ & Coriolis and centrifugal forces of the dual-arm manipulators \\
\hline$g_{D}$ & Gravitational forces of the dual-arm manipulators \\
\hline$\theta_{m d r}$ & Linearized dynamic parameters of the dual-arm manipulators \\
\hline$Y_{m d}()$. & Dynamic regression matrix of the dual-arm manipulators \\
\hline$M_{o}\left(x_{o}\right)$ & Inertial matrix of the grasped object \\
\hline$C_{o} \dot{x}_{o}$ & Vector of the object's Coriolis and centrifugal forces \\
\hline$g_{o}$ & Gravitational forces of the object \\
\hline$F_{o}$ & Resultant force acting on the object's COM by the two manipulators \\
\hline$F_{t}$ & Contact force exerted by the environment on the object's end tip \\
\hline $\mathcal{N}$ & Null-space matrix of $J_{o}^{T}\left(x_{o}\right)$ \\
\hline$F_{I}$ & Internal force in the null-space matrix of $J_{o}^{T}\left(x_{o}\right)$ \\
\hline $\mathcal{F}$ & Vector which describes the direction of the internal force \\
\hline$\lambda_{I}$ & Lagrange multiplier of $F_{I}$ \\
\hline$\lambda_{t}$ & Lagrange multiplier of $F_{t}$ \\
\hline$r_{o}$ & $\begin{array}{l}\text { Vector combined by three vectors from the object's COM to the corresponding contact } \\
\text { points expressed in the object frame }\end{array}$ \\
\hline$R_{o}$ & Rotation matrix from object frame to world frame \\
\hline$R\left(x_{o}\right)$ & Regression matrix of $R_{o} r_{o}$ \\
\hline$r$ & Linearized parameter vector of $R_{o} r_{o}$ \\
\hline$\varphi, v$ & Positive gain constants of the estimation method of object's COM \\
\hline$\Delta \lambda_{t}, \Delta \lambda_{I}$ & Tracking error of the contact force and internal force \\
\hline$\lambda_{t d}, \lambda_{I d}$ & Desired contact force and internal force \\
\hline$\Delta \lambda_{F t}, \Delta \lambda_{F I}$ & Integration of $\Delta \lambda_{t}$ and $\Delta \lambda_{I}$ \\
\hline$R_{t}$ & Rotation matrix defined by Eq. (58) \\
\hline$x_{t d}$ & Desired end tip position \\
\hline$\dot{x}_{t r}$ & Reference tip velocity defined by Eq. (25) \\
\hline
\end{tabular}




\begin{tabular}{ll}
\hline Symbol & Definition \\
\hline$\alpha, \beta$ & Positive constants in the definition of $\dot{x}_{t r}$ \\
$\dot{q}_{r}$ & Reference joint velocity defined by Eq. (26) \\
$\psi$ & Minus the gradient of a convex function for optimization \\
$N_{J_{o}^{\dagger}}$ & Null-space matrix of $J_{o}^{\dagger}$ \\
$\sigma_{i}$ & Singular value of the estimated Jacobian $\hat{J}_{D}$ \\
$\varepsilon$ & Pre-specified constant which defines the size of the singular region \\
$\lambda_{\text {max }}$ & Maximum of the damping factor \\
$s$ & Joint-space sliding variable defined by Eq. (29) \\
$\bar{s}_{O}$ & Auxiliary sliding variable defined by Eq. (31) \\
$\dot{\bar{x}}_{o r}$ & Auxiliary reference object velocity $J_{o}^{\dagger} J_{D} \dot{q}_{r}$ \\
$Y_{o d r}, \theta_{\text {odr }}$ & Regression matrix and parameters defined by Eq. (37) \\
$Y_{J o d}, \theta_{J o d}$ & Regression matrix and parameters defined by Eq. (38) \\
$Y_{f t}, \theta_{f t}$ & Regression matrix and parameters defined by Eq. (39) \\
$Y_{f I}, \theta_{f I}$ & Regression matrix and parameters defined by Eq. (40) \\
$\kappa, \gamma$ & Positive constant in Eqs. (26) and (44) \\
$K_{p}, K, L_{d}$, & Positive definite gain matrix with proper dimensions \\
$L_{f t}, L_{f I}, L_{k}$, & \\
$L_{J o d}$ &
\end{tabular}

\section{Supplementary materials}

Supplementary material associated with this article can be found, in the online version, at doi:10.1016/j.jfranklin.2017.09.015.

\section{References}

[1] R. Zollner, T. Asfour, R. Dillmann, Programming by demonstration: dual-arm manipulation tasks for humanoid robots, in: 2004 IEEE/RSJ International Conference on Intelligent Robots and Systems, 2004. Proceedings, 1, IEEE, 2004.

[2] J. Lee, P.H. Chang, R.S. Jamisola, Relative impedance control for dual-arm robots performing asymmetric bimanual tasks, IEEE Trans. Ind. Electron. 61 (7) (2014) 3786-3796.

[3] V. Lippiello, L. Villani, B. Siciliano, An open architecture for sensory feedback control of a dual-arm industrial robotic cell, Ind. Robot 34 (1) (2007) 46-53.

[4] K.G. Tzierakis, F.N. Koumboulis, Independent force and position control for cooperating manipulators, J. Franklin Inst. 340 (6) (2003) 435-460.

[5] R.C. Bonitz, T.C. Hsia, Internal force-based impedance control for cooperating manipulators, IEEE Trans. Rob. Autom. 12 (1) (1996) 78-89.

[6] S.A. Schneider, R.H. Cannon, Object impedance control for cooperative manipulation: theory and experimental results, IEEE Trans. Rob. Autom. 8 (3) (1992) 383-394.

[7] F. Caccavale, P. Chiacchio, A. Marino, L. Villani, Six-DOF impedance control of dual-arm cooperative manipulators, IEEE/ASME Trans. Mechatron. 13 (5) (2008) 576-586.

[8] Y. Ren, Y. Liu, M. Jin, H. Liu, Biomimetic object impedance control for dual-arm cooperative 7-DOF manipulators, Rob. Auton. Syst. 75 (2016) 273-287.

[9] C. Smith, Y. Karayiannidis, L. Nalpantidis, X. Gratal, P. Qi, D.V. Dimarogonas, D. Kragic, Dual arm manipulation-a survey, Rob. Auton. Syst. 60 (10) (2012) 1340-1353.

[10] J.T. Wen, K. Kreutz-Delgado, Motion and force control of multiple robotic manipulators, Automatica 28 (4) (1992) 729-743.

[11] M.W. Walker, Adaptive control of manipulators containing closed kinematic loops, IEEE Trans. Rob. Autom. 6 (1) (1990) 10-19. 
[12] J.H. Jean, L.C. Fu, Adaptive hybrid control strategies for constrained robots, IEEE Trans. Autom. Control 38 (4) (1993) 598-603.

[13] P. Pagilla, M. Tomizuka, Hybrid force/motion control of two arms carrying an object, in: American Control Conference, 1, IEEE, Baltimore, June 1994, pp. 195-199.

[14] W. Gueaieb, F. Karray, S. Al-Sharhan, A robust hybrid intelligent position/force control scheme for cooperative manipulators, IEEE/ASME Trans. Mechatron. 12 (2) (2007) 109-125.

[15] K.Y. Lian, C.S. Chiu, P. Liu, Semi-decentralized adaptive fuzzy control for cooperative multirobot systems with Hळ motion/internal force tracking performance, IEEE Trans. Syst. Man Cybern. Part B Cybern. 32 (3) (2002) 269-280.

[16] W.H. Zhu, Y.G. Xi, Z.J. Zhang, Z. Bien, J. De Schutter, Virtual decomposition based control for generalized high dimensional robotic systems with complicated structure, IEEE Trans. Rob. Autom. 13 (3) (1997) $411-436$.

[17] T. Yoshikawa, A. Sudou, Dynamic hybrid position/force control of robot manipulators-on-line estimation of unknown constraint, IEEE Trans. Rob. Autom. 9 (2) (1993) 220-226.

[18] M. Namvar, F. Aghili, Adaptive force-motion control of coordinated robots interacting with geometrically unknown environments, IEEE Trans. Rob. 21 (4) (2005) 678-694.

[19] A.C. Leite, F. Lizarralde, L. Hsu, Hybrid adaptive vision-force control for robot manipulators interacting with unknown surfaces, Int. J. Rob. Res. 28 (7) (2009) 911-926.

[20] F. Aghili, Adaptive control of manipulators forming closed kinematic chain with inaccurate kinematic model, IEEE/ASME Trans. Mechatron. 18 (5) (2013) 1544-1554.

[21] A. Jafari, J.H. Ryu, Independent force and position control for cooperating manipulators handling an unknown object and interacting with an unknown environment, J. Franklin Inst. 353 (4) (2016) 857-875.

[22] R. Monfaredi, S.M. Rezaei, A. Talebi, A new observer-based adaptive controller for cooperative handling of an unknown object, Robotica 34 (07) (2016) 1437-1463.

[23] C.C. Cheah, S.P. Hou, Y. Zhao, J.J.E. Slotine, Adaptive vision and force tracking control for robots with constraint uncertainty, IEEE/ASME Trans. Mechatron. 15 (3) (2010) 389-399.

[24] H. Wang, Y. Xie, Adaptive Jacobian position/force tracking control of free-flying manipulators, Rob. Auton. Syst. 57 (2) (2009) 173-181.

[25] Y. Zhao, C.C. Cheah, Neural network control of multifingered robot hands using visual feedback, IEEE Trans. Neural Networks 20 (5) (2009) 758-767.

[26] T. Wimböck, C. Ott, A. Albu-Schäffer, G. Hirzinger, Comparison of object-level grasp controllers for dynamic dexterous manipulation, Int. J. Rob. Res. 31 (1) (2012) 3-23.

[27] M.E. Abdallah, C.W. Wampler, R. Platt, Object impedance control using a closed-chain task definition, in: 2010 10th IEEE-RAS International Conference on Humanoid Robots, IEEE, Nashville, December 2010, pp. $269-274$.

[28] L. Chao, C.C. Cheah, J.-J.E. Slotine, Adaptive Jacobian tracking control of rigid-link electrically driven robots based on visual task-space information, Automatica 42 (9) (2006) 1491-1501.

[29] M.W. Spong, S. Hutchinson, M. Vidyasagar, Robot Modeling and Control, 3, Wiley, New York, 2006, pp. 303-304.

[30] B. Siciliano, L. Sciavicco, L. Villani, G. Oriolo, Robotics: Modelling, Planning and Control, Springer Science \& Business Media, 2010.

[31] I.D. Walker, R.A. Freeman, S.I. Marcus, Analysis of motion and internal loading of objects grasped by multiple cooperating manipulators, Int. J. Rob. Res. 10 (4) (1991) 396-409.

[32] J.H. Chung, B.J. Yi, W.K. Kim, Analysis of internal loading at multiple robotic systems, J. Mech. Sci. Technol. 19 (8) (2005) 1554-1567.

[33] S. Erhart, S. Hirche, Internal force analysis and load distribution for cooperative multi-robot manipulation, IEEE Trans. Rob. 31 (5) (2015) 1238-1243.

[34] Z. Li, J. Li, Y. Kang, Adaptive robust coordinated control of multiple mobile manipulators interacting with rigid environments, Automatica 46 (12) (2010) 2028-2034.

[35] X. Zhang, Matrix Analysis and Applications, second ed., Tsinghua and Springer Publishing House, Beijing, 2013, pp. 193-284.

[36] Y. Ren, Y. Zhou, Y. Liu, et al., Robust adaptive multi-task tracking control of redundant manipulators with dynamic and kinematic uncertainties and unknown disturbances, Adv. Rob. 31 (9) (2017) 482-495.

[37] S. Chiaverini, Singularity-robust task-priority redundancy resolution for real-time kinematic control of robot manipulators, IEEE Trans. Rob. Autom. 13 (3) (1997) 398-410.

[38] P.A. Ioannou, J. Sun, Robust Adaptive Control, Courier Corporation, New York, 2012, pp. 66-143.

[39] W. Gueaieb, S. Al-Sharhan, M. Bolic, Robust computationally efficient control of cooperative closed-chain manipulators with uncertain dynamics, Automatica 435 (2007) 842-851. 